\title{
Pooling Methods for Likelihood Ratio Tests in Multiply Imputed Data Sets
}

\author{
Simon Grund (DD ${ }^{1,2}$, Oliver Lüdtke (D) ${ }^{1,2}$, and Alexander Robitzsch (D) ${ }^{1,2}$ \\ ${ }^{1}$ Leibniz Institute for Science and Mathematics Education \\ ${ }^{2}$ Centre for International Student Assessment
}

\begin{abstract}
Author Note
Simon Grund (D) 0000-0002-1290-8986

Oliver Lüdtke (D) 0000-0001-9744-3059

Alexander Robitzsch (D) 0000-0002-8226-3132

All additional files concerning this article, including scripts, data, and the supplemental materials are available at: https://osf.io/u9s4k. A copy of this manuscript was posted on the PsyArXiv preprint server: https://doi.org/10.31234/osf.io/d459g.
\end{abstract}

Correspondence concerning this article should be addressed to Simon Grund, Leibniz Institute for Science and Mathematics Education, 24118 Kiel, Germany; (+49)-431-880-5653; grund@leibniz-ipn.de. 


\begin{abstract}
Likelihood ratio tests (LRTs) are a popular tool for comparing statistical models. However, missing data are also common in empirical research, and multiple imputation (MI) is often used to deal with them. In multiply imputed data, there are multiple options for conducting LRTs, and new methods are still being proposed. In this article, we compare all available methods in multiple simulations covering applications in linear regression, generalized linear models, and structural equation modeling (SEM). In addition, we implemented these methods in an R package, and we illustrate its application in an example analysis concerned with the investigation of measurement invariance.
\end{abstract}

Keywords: missing data, multiple imputation, model comparison, likelihood ratio test 


\section{Pooling Methods for Likelihood Ratio Tests in Multiply Imputed Data Sets}

Model comparison is one of the most common tasks in empirical research. By comparing models, researchers evaluate how well each model from a set of candidate models fits the observed data and whether restrictions placed on the model parameters change the fit of the model in a meaningful way. To this end, researchers often use multiparameter tests such as the Wald test, the likelihood ratio test (LRT), or the score test (for an overview, see Buse, 1982; Cox, 2006). In practice, model comparisons are often complicated by missing data, for example, due to unintended nonresponse or planned missingness (Graham et al., 2006; Rhemtulla et al., 2014; Rhemtulla \& Hancock, 2016). Multiple imputation (MI) is one of the most common methods for dealing with missing data (Schafer \& Graham, 2002) as it is designed to replace missing values by creating multiple copies of the data, each with the missing values "filled in" with plausible replacements (Rubin, 1987). These data sets are then analyzed separately with conventional statistical methods, and the results are pooled to obtain a final set of parameter estimates and inferences.

The use of MI can be advantageous when there are multiple models of interest because it separates the treatment of missing data from the analysis. This ensures that the same assumptions about the missing data underlie the estimation and comparison of all models. On the other hand, the model comparison itself can become challenging when using MI because it requires the results to be pooled across the imputed data sets. Pooling methods for various types of analyses have been proposed in the missing data literature (for an overview, see Reiter and Raghunathan, 2007). The main focus of this article is on model comparisons with LRTs, but similar methods exist for Wald and score tests (Li, Raghunathan, et al., 1991; Mansolf et al., 2020). Several methods have been proposed for pooling LRTs (e.g., see Schafer, 1997), and new methods are still being

developed (Chan \& Meng, 2022). However, their properties are still poorly understood, and little is known about which methods are the most reliable for pooling LRTs in typical applications in psychology and related fields.

The present article is aimed at reducing this gap by evaluating the methods currently available for pooling LRTs in MI and by facilitating their application with an R package in which 
all the methods are implemented. The article is structured as follows. First, we briefly review the statistical foundation of LRTs in complete data before we outline the methods that have been proposed so far for pooling LRTs in MI. In this context, we also discuss the theoretical and practical advantages and disadvantages of each method. Then, we present the results of three simulation studies in which we evaluated the performance of each method in applications typical for regression analysis, analyses with generalized linear models (GLMs), and structural equation modeling (SEM). Finally, we illustrate the application of theses methods with an example analysis using SEM and the R package mitml (Grund, Robitzsch, et al., 2021).

\section{Complete-Data LRTs}

In complete data sets, an LRT is performed by comparing two models with respect to the $\log$-likelihood of the data, $\ell(\theta)=\log f(Y \mid \theta)$, given a set of model parameters $\theta$. Specifically, the LRT is performed by calculating

$$
d=-2\left[\ell\left(\hat{\theta}_{0}\right)-\ell(\hat{\theta})\right]
$$

where $\hat{\theta}$ denotes the estimated parameters under the full model, and $\hat{\theta}_{0}$ denotes the estimated parameters under a restricted (or $n u l l$ ) model. The LRT statistic $d$ (also known as the deviance) is typically compared with a $\chi^{2}$ distribution with $k$ degrees of freedom, where $k$ is the number of parameters being tested, that is, the number of restricted elements in $\theta_{0}$ (for a general treatment, see Held \& Bové, 2014).

\section{Pooling Methods for LRTs}

In MI, each of the $m$ imputed data sets is analyzed separately. This results in $m$ sets of parameter estimates for the full model, $\hat{\theta}^{(1)}, \ldots, \hat{\theta}^{(m)}$, and the null model, $\hat{\theta}_{0}^{(1)}, \ldots, \hat{\theta}_{0}^{(m)}$. The estimates themselves can easily be pooled simply by using Rubin's (1987) rules. By contrast, it is a lot less straightforward to pool the LRT statistics $d^{(1)}, \ldots, d^{(m)}$, which compare the $\log$-likelihood of the imputed data under the full model, $\ell_{1}\left(\hat{\theta}^{(1)}\right), \ldots, \ell_{m}\left(\hat{\theta}^{(m)}\right)$, with the loglikelihood of the same set of imputed data under the null model, $\ell_{1}\left(\hat{\theta}_{0}^{(1)}\right), \ldots, \ell_{m}\left(\hat{\theta}_{0}^{(m)}\right)$. In the following, we briefly describe the different methods that have been proposed for this task thus far. 


\section{Pooled $\chi^{2}$ Statistics $\left(D_{2}\right)$}

Li, Meng, et al. (1991) proposed that LRTs be pooled by pooling the corresponding $\chi^{2}$ statistics (or equivalently the $p$ values) that are obtained from the LRTs in each of the imputed data sets. This method has been labeled $D_{2}$ in the missing data literature (Schafer, 1997). The $D_{2}$ statistic is calculated as

$$
D_{2}=\frac{\bar{d}-\frac{k(m-1)}{m+1} r_{2}}{k\left(1+r_{2}\right)}
$$

where $\bar{d}$ is the average LRT statistic across the imputed data sets, and $r_{2}$ is an estimate of the average relative increase in variance (ARIV) due to nonresponse:

$$
r_{2}=\left(1+\frac{1}{m}\right)\left[\frac{1}{m-1} \sum_{l=1}^{m}\left(\sqrt{d^{(l)}}-\overline{\sqrt{d}}\right)^{2}\right]
$$

with $\overline{\sqrt{d}}=m^{-1} \sum_{l=1}^{m} \sqrt{d^{(l)}}$. Broadly speaking, the ARIV represents the average increase in the sampling variance in the parameters being tested that is due to missing data. The ARIV is conceptually related to the fraction of missing information (FMI), which describes how much of the information that the data provide about the parameters is lost due to missing data (see also Enders, 2010; Rubin, 1987). $D_{2}$ requires that the FMI of the parameters that are being tested is approximately equal, so that their increases in variance can be meaningfully summarized by the ARIV. The $D_{2}$ statistic is typically compared with an $F$ distribution that has $k$ numerator and $\nu_{2}$ denominator degrees of freedom:

$$
\nu_{2}=k^{-3 / m}(m-1)\left(1+r_{2}^{-1}\right)^{2} .
$$

From a theoretical perspective, the $F$ distribution used in $D_{2}$ corresponds to the $\chi^{2}$ distribution that is used for LRTs in complete data but includes a finite number of denominator degrees of freedom $\left(\nu_{2}\right)$ to account for the finite number of imputations that is used in MI. ${ }^{1}$

\footnotetext{
${ }^{1}$ Despite the use of the $F$ distribution, the pooling methods are still based on the assumption that the sample is large enough for the corresponding complete-data LRT statistic to follow a $\chi^{2}$ distribution. This is contrast with pooling methods for other quantities (e.g., Li, Raghunathan, et al., 1991), which can be adjusted to accommodate smaller samples (Barnard \& Rubin, 1999; Reiter, 2007).
} 
Figure 1 provides a visual illustration of $D_{2}$ as well as the other pooling methods that we are considering here. The main principle of $D_{2}$ is to evaluate the LRT statistics separately in each data set and to pool the results directly into an average value that is adjusted in accordance with the ARIV to reflect the added uncertainty that is due to missing data. As a result, calculating $D_{2}$ requires only the $\chi^{2}$ statistics (or equivalently the $p$ values) from the $m$ imputed data sets.

\section{Pooled LRTs $\left(D_{3}\right)$}

To provide an alternative method for pooling LRTs in multiply imputed data, Meng and Rubin (1992) introduced the $D_{3}$ statistic. This statistic is calculated as

$$
D_{3}=\frac{\tilde{d}}{k\left(1+r_{3}\right)}
$$

where $\tilde{d}$ is the average LRT statistic evaluated at the pooled parameter estimates (e.g., with the pooled values obtained through Rubin's rules), and $r_{3}$ is another estimate of the ARIV:

$$
r_{3}=\frac{m+1}{k(m-1)}(\bar{d}-\tilde{d})
$$

Like $D_{2}$, the $D_{3}$ statistic is based on the assumption that the FMI of the parameters being tested is approximately equal. $D_{3}$ can be compared with an $F$ distribution with $k$ numerator and $\nu_{3}$ denominator degrees of freedom:

$$
\nu_{3}=\left\{\begin{array}{ll}
4+(t-4)\left[1+\left(1-2 t^{-1}\right) r_{3}^{-1}\right]^{2} & \text { if } t=k(m-1)>4 \\
t\left(1+k^{-1}\right)\left(1+r_{3}^{-1}\right)^{2} / 2 & \text { otherwise }
\end{array} .\right.
$$

Figure 1 again illustrates the main principle of $D_{3}$. In contrast to $D_{2}$, the $D_{3}$ statistic requires the LRT statistic to be evaluated twice for each of the imputed data sets: once at the parameter

estimates in that particular data set (i.e., $\theta^{(l)}$ and $\theta_{0}^{(l)}$ for the $l$ th data set); and once with the parameters fixed to their pooled values (i.e., $\bar{\theta}$ and $\bar{\theta}_{0}$ ).

\section{Pooled LRTs with “Stacked” Data $\left(D_{4}\right)$}

Recently, Chan and Meng (2022) proposed yet another method for pooling LRTs that 
relies on the construction of a "stacked" data set, that is, a combined data set with all imputed data sets stacked on top of each other (see also Chan and Meng, 2019; for some earlier methods based on stacked data, see also Lang and Little, 2014, Wood et al., 2008). In keeping with the previous literature (e.g., Schafer, 1997), we refer to this method as $D_{4}$. Let $\ell_{S}\left(\theta_{S}\right)=m^{-1} \log f\left(Y_{S} \mid \theta_{S}\right)$ denote the log-likelihood of the stacked data. Then $D_{4}$ is calculated as

$$
D_{4}=\frac{\hat{d}_{S}}{k\left(1+r_{4}\right)},
$$

where $\hat{d}_{S}=-2\left[\ell_{S}\left(\hat{\theta}_{0, S}\right)-\ell_{S}\left(\hat{\theta}_{S}\right)\right]$ is the test statistic of the LRT that is obtained by fitting and comparing the full and the null model in the stacked data, and $r_{4}$ is yet another estimate of the ARIV,

$$
r_{4}=\frac{m+1}{k(m-1)}\left(\bar{d}-\hat{d}_{S}\right)
$$

Like $D_{2}$ and $D_{3}$, the $D_{4}$ statistic is again based on the assumption that the FMI of the parameters being tested is approximately equal. Because $r_{4}$ can take on negative values (similar to $r_{3}$ ), Chan and Meng (2022) recommended that the estimator of the ARIV in Equation 9 be replaced by $r_{4}^{+}=$ $\max \left(0, r_{4}\right)$ to ensure that the resulting expression is positive. ${ }^{2}$ The $D_{4}$ statistic can be compared with an $F$ distribution that has $k$ numerator and $\nu_{4}$ denominator degrees of freedom:

$$
\nu_{4}=k(m-1)\left[1+\left(r_{4}^{+}\right)^{-1}\right]^{2}
$$

Figure 1 provides an illustration of $D_{4}$. In contrast to $D_{3}$, the $D_{4}$ statistic relies on the stacked data set instead of pooled parameter estimates for pooling the LRT. As a result, calculating $D_{4}$ requires only the evaluation of the LRT statistic in the stacked data in addition to the $m$ individual LRT statistics obtained from the imputed data sets.

\footnotetext{
${ }^{2}$ In addition to the $D_{4}$ method as presented here, Chan and Meng (2022) also propose a "robust" version of $D_{4}$, which is based on a different (and more robust) estimate of the ARIV but requires the much stronger assumption that the relative increase in variance is equal for all parameters in the full model (i.e., not only the parameters that are being tested). For simplicity's sake, we focus on the non-robust version of $D_{4}$. However, interested readers can find additional details about and simulation results on the performance of the robust and non-robust versions of $D_{4}$ in Supplement A.
} 


\section{Comparison of Pooling Methods for LRTs}

The pooling methods presented above have different advantages and disadvantages. From a statistical point of view, $D_{2}$ has been criticized, because its sole reliance on the $\chi^{2}$ values from the LRTs in the imputed data sets constitutes an inefficient use of the information available about the models that are being compared (Li, Meng, et al., 1991; see also Enders, 2010; Schafer, 1997; van Buuren, 2018). This is in contrast to methods such as $D_{3}$ and $D_{4}$, which incorporate additional information about the model comparison through the pooled parameter estimates and the stacked data, respectively. Consequently, Li, Meng, et al. (1991) have found that $D_{2}$ can be either too liberal or too conservative depending on the number of parameters that are being tested and the FMI. Grund et al. (2016), Liu and Sriutaisuk (2020), and van Ginkel and Kroonenberg (2021) replicated these findings but also showed that $D_{2}$ can be fairly robust if the number of imputations is sufficiently large and the FMI is not too extreme (see also Liu \& Sriutaisuk, 2021; Liu et al., 2021). From a practical perspective, $D_{2}$ can be an attractive option because it is easy to compute, and the required $\chi^{2}$ values are routinely provided by statistical software.

The $D_{3}$ statistic has often been recommended as a more reliable method for pooling LRTs

in MI (Meng and Rubin, 1992; see also Enders, 2010; Schafer, 1997). Previous studies have found that $D_{3}$ usually works well but also that it tends to be slightly conservative and can suffer from low power, especially in conditions with large FMI (e.g., Grund et al., 2016; Liu \& Enders, 2017; van Ginkel \& Kroonenberg, 2021). However, $D_{3}$ has also been criticized because it is not invariant to the parameterization of the models that are being compared (e.g., Schafer, 1997). Specifically, because computing $D_{3}$ requires a re-evaluation of the likelihood function at the pooled values of the estimated parameters, its exact value depends on what parameterization is used to pool the parameter estimates. For example, if the goal is to compare two regression models, $D_{3}$ can be implemented by pooling either the residual variance $\left(\sigma^{2}\right)$ or standard deviation $(\sigma)$ and will return different values depending on which parameterization is used. In addition, using $D_{3}$ in practice can be difficult because it requires re-evaluating the likelihood function at pooled values of the model parameters, and such a low-level access to the likelihood function is 
often not provided by statistical software (Chan \& Meng, 2022).

Finally, $D_{4}$ has recently been proposed as another alternative with multiple improvements over $D_{3}$ (see Chan \& Meng, 2022). Specifically, $D_{4}$ is invariant to the parameterization of the models that are being compared. In addition, Chan and Meng (2022) argued that the performance of $D_{4}$ should be at least as good as (or better than) $D_{3}$. From a practical point of view, $D_{4}$ is a promising alternative because it requires only the construction of a stacked data set that is then analyzed with standard methods without the need to re-evaluate the likelihood at user-defined values. As a result, $D_{4}$ can be implemented more easily, both in statistical software and by researchers who are proficient with statistical modeling environments such as $\mathrm{R}$ ( $\mathrm{R}$ Core Team, 2021). However, to date, there have been no software packages that implement $D_{4}$, and no studies that have evaluated $D_{4}$ in comparison with other methods in applications that are common in psychology and related fields (however, see Chan \& Meng, 2022).

There are multiple software packages that implement one or more pooling methods for LRTs with support for different model types. For example, software that implements $D_{2}$ includes the R packages mi ce (van Buuren \& Groothuis-Oudshoorn, 2011), mi ceadds (Robitzsch et al., 2021), mitml (Grund, Robitzsch, et al., 2021), and semTools (Jorgensen et al., 2021). D $D_{3}$ can be used with the R packages mice (van Buuren \& Groothuis-Oudshoorn, 2011), mitml (Grund, Robitzsch, et al., 2021), and semTools (Jorgensen et al., 2021) as well as with the commercial software packages Mplus (Asparouhov \& Muthén, 2008), Stata (Medeiros, 2008), and SAS (Mistler, 2013). Finally, $D_{4}$ is implemented in the R package mitml (Grund, Robitzsch, et al., 2021).

In the following, we present the results of three simulation studies in which we compared the performance of these pooling methods in three different scenarios that represent common applications of LRTs in psychology and the social sciences with varying complexity: linear regression (Study 1), logistic regression (Study 2), and structural equation modeling (SEM, Study 3). The computer code needed to run the simulation studies is provided on the OSF project page (https://osf.io/u9s4k; Grund, Lüdtke, et al., 2021). 


\section{Study 1: Linear Regression}

\section{Data Generation}

In Study 1, we were interested in applications of the LRT for comparing models in the context of regression analyses. The data were generated from a linear regression model with a standardized outcome variable $y$ and $k$ standardized, normally distributed predictor variables $x_{j}$ $(j=1, \ldots, k)$. For person $i(i=1, \ldots, n)$,

$$
y_{i}=\beta_{0}+\sum_{j=1}^{k} \beta_{j} x_{j i}+e_{i}, \quad e_{i} \sim N\left(0, \sigma^{2}\right),
$$

where $\beta_{j}$ denotes the regression coefficient for the $j$ th predictor variable, and $\sigma^{2}$ denotes the residual variance. The predictors were correlated according to some correlation $\rho$ and had identical regression coefficients $\left(\beta_{j}=\beta\right)$. The value of the regression coefficients was chosen in such a way that a certain amount of variance $\left(R^{2}\right)$ was explained by the predictor variables (for a previous use of this design, see also Liu \& Enders, 2017).

To introduce missing values in the predictor variables, we divided the predictor variables $x_{j}$ up into two halves $(h=1,2)$. For each half, we generated missing values in accordance with the following linear model:

$$
r_{i}^{(h)}=\gamma_{0}+\gamma_{1}\left(\frac{y_{i}-\bar{y}}{S D_{y}}\right)+u_{i}^{(h)}, \quad u_{i}^{(h)} \sim N\left(0,1-\gamma_{1}^{2}\right),
$$

where $\gamma_{0}$ is a quantile of the standard normal distribution that controls the probability of missing data (e.g., $\gamma_{0}=-0.84$ for $20 \%$ missing data), and $\gamma_{1}$ determined if the data were missing completely at random (MCAR, if $\gamma_{1}=0$ ) or missing at random (MAR, if $\gamma_{1} \neq 0$; see Rubin, 1987). For each half and each person, we set all the predictors in this half to missing when the corresponding $r_{i}^{(h)}<0$.

\section{Simulated Conditions}

We varied the sample size $(n=50,100,200,500)$, the number of predictor variables $(k=$ $2,4,6)$, the proportion of variance explained by the predictor variables $\left(R^{2}=0, .02, .13, .26\right)$, the 
percentage of missing data per predictor $(p=10 \%, 20 \%, 30 \%)$, and whether the data were MCAR or MAR $\left(\gamma_{1}=0, .70\right)$. The FMIs that were implied by this design were equal for the regression coefficients $\beta_{j}$ and depended on the simulated $R^{2}$, the number of predictors, the percentage of missing data, and the missing data mechanism. Each condition was replicated between 1,000 and 3,000 times depending on the sample size. ${ }^{3}$

\section{Treatment of Missing Data and Analyses}

The missing data were imputed with the R package mice (van Buuren \& GroothuisOudshoorn, 2011). In line with current recommendations in the missing data literature (e.g., Bodner, 2008; Graham et al., 2007; von Hippel, 2020), we generated 100 imputed data sets, each after 20 iterations of the imputation algorithm. The model of interest was the regression model in Equation 11, and we used the LRT to compare this model with a restricted model that included only the intercept (i.e., no predictor variables). In this context, applying the LRT is equivalent to simultaneously testing all regression coefficients against zero. To implement the LRT for the imputed data, we used the methods outlined above:

$D_{2}:$ pooled $\chi^{2}$ values based on separate LRTs (Li, Meng, et al., 1991)

$D_{3}:$ pooled LRT (Meng \& Rubin, 1992)

$D_{4}:$ pooled LRT based on "stacked" data (Chan \& Meng, 2022)

To provide a means of comparison, we also applied the LRT to the complete data (CD) and after listwise deletion (LD), whereby all cases with missing data were removed from the analysis.

Finally, to provide an additional means of comparison, we also conducted the LRT after handling the missing data with full-information maximum likelihood (FIML; see Arbuckle, 1996) in the R package lavaan (Rosseel, 2012).

\footnotetext{
${ }^{3}$ Conditions with small samples $(n=50)$ were replicated 3,000 times, those with moderate samples $(n=100) 2,000$ times, those with large sample $(n=200) 1,500$ times, and those with very large samples $(n=500) 1,000$ times. As a result, the Monte Carlo SEs for the Type 1 error rates ranged from $0.4 \%$ to $0.7 \%$, assuming that the actual Type 1 error rates were close to the nominal value of $5 \%$.
} 
Evaluation Criteria. All methods were evaluated in terms of their Type 1 error rates (at the $\alpha=5 \%$ level) in conditions with $R^{2}=0$ and their statistical power in conditions with nonzero effects $\left(R^{2}>0\right)$. In this context, we considered Type 1 error rates between $2.5 \%$ and $7.5 \%$ to be acceptable (Bradley, 1978). Furthermore, to facilitate the interpretation of the results, we also calculated the empirical FMI about the LRT in each simulated condition by comparing the variance of the estimated regression coefficients in MI and CD (see Savalei and Rhemtulla, 2012). Specifically, let $\operatorname{RIV}_{j}=\left[\operatorname{Var}\left(\hat{\beta}_{j r}^{M I}\right)-\operatorname{Var}\left(\hat{\beta}_{j r}^{C D}\right)\right] / \operatorname{Var}\left(\hat{\beta}_{j r}^{C D}\right)$ denote the relative increase in variance for each regression coefficient $(j=1, \ldots, k)$ across the replications $(r=1, \ldots, s)$ of each simulated condition. The empirical FMI is then given by:

$$
\mathrm{FMI}=\frac{\frac{1}{k} \sum_{j=1}^{k} \mathrm{RIV}_{j}}{1+\frac{1}{k} \sum_{j=1}^{k} \mathrm{RIV}_{j}}
$$

The empirical FMI takes values between 0 and 1 for each simulated condition and can be interpreted as a descriptive measure for the effective loss of information about the parameters being tested - and thus the LRT — that was due to missing data in the respective condition.

\section{Results}

Due to the large number of results, we focus on the conditions with MAR data and discuss the results in MCAR data only when needed. The full set of results is provided in Supplement C in the online supplemental materials.

Type 1 Error Rates. In conditions with only a few missing values (10\%), all procedures had Type 1 error rates close to the nominal value of 5\% in most cases. The Type 1 error rates of all the procedures in the conditions with MAR data are summarized in Table 1 . The only exceptions to this rule were with respect to CD, LD, FIML, and $D_{2}$, which showed a noticeable increase in Type 1 errors in conditions with small samples $(n=50)$ and many predictors $(k=6)$. Because these problems were also present in CD, this primarily reflected the behavior of the LRT in small samples. By contrast, $D_{3}$ and $D_{4}$ tended to show slightly lower rates of Type 1 errors in comparison with $\mathrm{CD}$ and maintained the nominal level throughout. 
In conditions with large amounts of missing data (30\%), the differences between the procedures were more pronounced. Specifically, with LD, FIML, and $D_{2}$, the Type 1 error rates were often larger, reflecting more liberal statistical inferences, whereas those obtained with $D_{3}$ and $D_{4}$ were smaller, reflecting that these procedures were more conservative. These differences were especially large in conditions with small samples $(n=50)$ and many predictors $(k=6)$, where the procedures led to Type 1 error rates that were well above ( $11.0 \%$ for LD, $10.3 \%$ for FIML, and $16.5 \%$ for $\left.D_{2}\right)$ or below (1.2\% for $D_{3}$ and $1.9 \%$ for $\left.D_{4}\right)$ the nominal value. In conditions with larger samples, the Type 1 error rates obtained with LD, FIML, $D_{3}$, and $D_{4}$ again came close to the nominal value, even in these otherwise extreme conditions; however, those obtained with $D_{2}$ sometimes remained slightly too high. The results in the conditions with MCAR data followed the same pattern, but the differences between the procedures tended to be slightly smaller, which can be regarded as an effect of the smaller FMI in these conditions.

Power. For the statistical power to detect nonzero effects, the results closely matched those for the Type 1 error rates and are summarized in Figure 2 for conditions with a medium effect size $\left(R^{2}=.13\right)$ and MAR data. Specifically, the power tended to be higher for FIML and $D_{2}$, which reflected a more liberal behavior, and slightly lower for $D_{3}$ and $D_{4}$, which reflected a more conservative behavior. For LD, the power was usually lower than the other methods except for conditions with small samples $(n=50)$, a larger number of parameters $(k>4)$ and at least moderate amounts of missing data (20\% or more), where LD sometimes had more power than $D_{3}$, and $D_{4}$. The differences between the procedures were more pronounced in conditions with many predictors $(k=6)$ and more missing data $(30 \%)$, and tended to be smaller in less extreme conditions. Naturally, all procedures exhibited lower statistical power than CD. The overall pattern of results was similar for small $\left(R^{2}=.02\right)$ and large effects $\left(R^{2}=.26\right.$; see Supplement $\left.\mathrm{C}\right)$. However, the differences between the procedures were less pronounced in these conditions because the statistical power was generally much lower or higher regardless of which method was used to conduct the LRT. The results in the conditions with MCAR data again followed the same pattern (see Supplement C). 


\section{Effects of the Number of Imputations}

To investigate the potential impact of the number of imputations on model comparisons in MI, we repeated selected conditions from Study 1 with a different number of imputations. For this purpose, we chose a fixed sample size of $n=100$ with a fixed percentage of MAR data (20\%) and varied the number of predictors $(k=2,4,6)$, the amount of variance explained $\left(R^{2}=0, .13\right)$, and the number of imputations $(m=3,5,10,20,50,100,200,500,1,000)$. These conditions were replicated 2,000 times, and the results are summarized in Figure 3. The Type 1 error rates $\left(R^{2}=\right.$ 0 ) were not strongly affected by the number of imputations but showed a slight tendency for $D_{2}$ to be more conservative with fewer imputations and for $D_{3}$ and $D_{4}$ to be more liberal than they were when the number of imputations was large. The power $\left(R^{2}=.13\right)$ tended to increase with more imputations, especially for $D_{2}$, which tended to have a much lower power than $D_{3}$ and $D_{4}$ when the number of imputations was low $(m<20)$. These difference were stronger when more parameters were tested. Overall, these results illustrate the importance of the number of imputations and suggest that at least 20 imputations should be used to obtain stable results.

\section{Study 2: Logistic Regression}

\section{Data Generation}

In Study 2, we were interested in using the LRT to compare models in the context of generalized linear models (GLMs). To this end, we generated data from a logistic regression model with a binary outcome variable $y$ and $k$ standardized predictor variables $x_{j}(j=1, \ldots, k)$. For person $i(i=1, \ldots, n)$,

$$
\log \left(\frac{P\left(y_{i}=1 \mid X=x_{i}\right)}{1-P\left(y_{i}=1 \mid X=x_{i}\right)}\right)=\beta_{0}+\sum_{j=1}^{k} \beta_{j} x_{i j},
$$

where all predictors were correlated in an identical manner as before. The coefficients were chosen in such a way that the predictors explained a certain amount of variance $\left(R^{2}\right)$ in the latent response propensity underlying the logistic regression model (i.e., by simulating the binary responses on the basis of a latent response propensity $y_{i}^{*}$ with residuals that followed a logistic distribution with variance $\pi^{2} / 3$; for details, see Agresti, 2007). Missing data were induced as before. 


\section{Simulated Conditions, Imputation, and Analysis}

To accommodate the larger sample size requirements of GLMs, we increased the sample size $(n=100,200,500,1,000)$ in comparison with the previous study and varied the number of predictor variables $(k=2,4,6)$, the proportion of variance explained by the predictor variables $\left(R^{2}=0, .02, .13, .26\right)$, the percentage of missing data $(p=10 \%, 20 \%, 30 \%)$, and whether the data were MCAR or MAR. Each condition was replicated between 1,000 and 3,000 times as before.

The missing data were imputed in the same manner as before. ${ }^{4}$ The model of interest was the logistic regression model in Equation 14, and we again used an LRT to compare this model with a restricted model that included only an intercept. We used the same methods as before to pool the LRTs in the imputed data and the same procedures for comparison purposes. However, because the analysis featured a categorical outcome, we used Mplus (Muthén \& Muthén, 2012) to implement FIML. All methods were evaluated with respect to Type 1 error rates and power.

\section{Results}

The results for the Type 1 error rates and the power in the conditions with MAR data are summarized in Table 2 and Figure 4, respectively. Similar to the previous study, all procedures provided acceptable Type 1 error rates except for some of the conditions with small samples $(n=$ 100), many predictors $(k \geq 4)$, and a large percentage of missing values (30\%). In these conditions, LD and $D_{2}$ showed elevated Type 1 error rates, whereas FIML, $D_{3}$ and $D_{4}$ showed acceptable Type 1 error rates that were only slightly above or below the nominal value. As before, the Type 1 error rates obtained with LD, FIML, $D_{3}$, and $D_{4}$ approached the nominal value in conditions with larger samples, and this was also true for $D_{2}$. The results for the statistical power again followed a similar pattern, whereby the power tended to be slightly higher for FIML and $D_{2}$ and slightly lower for $D_{3}$ and $D_{4}$. Owing to the larger sample size in this study, the statistical power of LD was usually lower than that of the other methods. These differences were most

\footnotetext{
${ }^{4}$ In practice, missing data can also occur in the binary outcome variable and should be addressed with imputation methods for categorical data. In the fully conditional specification (FCS) approach used in mi ce, methods that are based on logistic regression can be used (van Buuren et al., 2006). In addition, a joint modeling approach (JM) can be used, in which binary variables are represented by (latent) continuous variables (Quartagno \& Carpenter, 2019).
} 
pronounced in conditions with many predictors $(k=6)$ and a large percentage missing data $(30 \%)$; in less extreme conditions, these differences were still visible but generally smaller. The results in the conditions with MCAR data again followed the same pattern, but the differences between the procedures tended to be slightly smaller (see Supplement C).

\section{Study 3: SEM}

\section{Data Generation}

In Study 3, we were interested in using the LRT for model comparisons in the context of SEM. The data were generated from a two-factor confirmatory factor analysis (CFA) model with $k$ items per factor and correlated residuals (or uniquenesses) between the corresponding items of each factor, as shown in Figure 5. This model is a common choice in applications of CFA in longitudinal research or in studies with multiple ratings of the same construct (e.g., self- vs. other ratings), where the assumption of independent unique factors is often not justified (e.g., Jöreskog, 1979; Little, 2013; Marsh, 1989). For identification, the factors were standardized with a mean of zero and a variance of one.

We introduced missing values into all the second-factor items on the basis of the firstfactor items. To this end, we divided the second-factor items up into two halves and generated missing values in accordance with the following model:

$$
r_{i}^{(h)}=\gamma_{0}+\gamma_{1}\left(\frac{\bar{y}_{i, 1 \bullet}-\bar{y}_{1 \bullet}}{S D_{y_{1} \bullet}}\right)+u_{i}^{(h)}, \quad u_{i}^{(h)} \sim N\left(0,1-\gamma_{1}^{2}\right),
$$

where $\bar{y}_{i, 1}$ is the mean of the first-factor items for person $i$, and $\bar{y}_{1} \bullet$ is the mean of the $\bar{y}_{i, 1}$ across persons. For each half and each person, we set all the second-factor items in this half to missing when the corresponding $r_{i}^{(h)}<0$.

\section{Simulated Conditions, Imputation, and Analysis}

We varied the sample size $(n=100,200,500,1,000)$, the number of items per factor $(k=$ $4,6,8)$, the correlation between the residuals $(\rho=0, .10, .20)$, and the percentage of missing data 
$(p=10 \%, 20 \%, 30 \%)$. We fixed the correlation between the two factors to $\phi=.50$. The factor loadings $(\boldsymbol{\Lambda})$ were chosen such that (a) the loadings for the corresponding items on each factor were equal and (b) their values were evenly distributed across the range of 0.70 to 0.95 as shown in Table 3. This corresponds to item reliabilities of roughly between .50 and .90 . In contrast with the previous studies, the FMIs for the residual correlations that were implied by this design differed slightly due to the differences in the item loadings and also depended on the simulated correlations between the factors $(\phi)$ and items $(\rho)$, the number of items per factor, the percentage of missing data, and the missing data mechanism. Each condition was replicated between 1,000 and 3,000 times as before.

Missing data were imputed in the same manner as before. To this end, we generated 100 imputations but increased the number of iterations per imputation to 50. The model of interest was the two-dimensional CFA model in Figure 5, and we used an LRT to compare this model with a restricted model in which the correlations between the residuals were fixed to zero. In this context, the LRT is used as a test for the presence or absence of correlated unique factors between corresponding items. Both models were estimated with standard maximum likelihood methods using the R package lavaan (Rosseel, 2012). To pool the LRT in the imputed data, we used the same methods as before. In addition, we used lavaan to implement FIML.

\section{Results}

Type 1 Error Rates. The results for the Type 1 error rates $(\rho=0)$ are summarized in Table 4 for conditions with MAR data. Overall, all procedures showed acceptable Type 1 error rates except for some of the conditions in which the sample size was small $(n=100)$ or the amount of missing data was large (30\%). In these conditions, the Type 1 error rates were sometimes too high for LD and FIML and too low for $D_{3}$ and $D_{4}$. However, these difference tended to be smaller than in the previous studies, which may reflect the fact that the FMI implied by the simulation design were somewhat lower. In contrast to the previous studies, $D_{2}$ showed Type 1 error rates that were close to the nominal value even in smaller samples. Similar to the 
previous studies, the Type 1 error rates obtained with LD, FIML, $D_{3}$, and $D_{4}$ came close to the nominal value as the sample size increased. The results in the conditions with MCAR data followed roughly the same pattern.

Power. The results for the statistical power to detect a nonzero correlation between the residuals are summarized in Figure 6 for conditions with weakly correlated residuals $(\rho=.10)$ and MAR data. In line with the results for the Type 1 error rates, the statistical power tended to be slightly higher for FIML than for $D_{3}$ and $D_{4}$. The power obtained with $D_{2}$ was usually lower than for FIML but higher than for $D_{3}$ and $D_{4}$. Finally, LD tended to have a slightly higher power than $D_{3}$ and $D_{4}$ in smaller samples $(n \leq 200)$ but lower power in larger samples $(n>200)$. However, these differences were relatively small, especially in larger samples $(n \geq 500)$, and they were most pronounced in conditions with the smallest sample sizes $(n=100)$, the largest number of items $(k=8)$, and the largest amount of missing data $(30 \%)$. Because conditions with a larger number of items also allowed for a more reliable measurement of the latent variables, the power was usually slightly higher in conditions with many $(k=8)$ versus only a few $(k=4)$ items, regardless of which method was used to conduct the LRT. The results in the conditions with MCAR data again followed a similar pattern.

\section{Example Analysis}

To illustrate the use of the pooling methods for LRTs, we used data from the EIKA study, a multi-cohort study conducted in Bremen, Germany, that investigated the psychological and socioeconomic antecedents of academic achievement in disadvantaged youths (EIKA, 2006). The data included self- and parent ratings of the Big Five personality traits for 943 ninth-grade students as well as information about their sex, cognitive ability, and reading and math proficiency. For simplicity, we focused only on extraversion. In this example, we aimed to investigate the extent to which measurement invariance held for the self- and parent ratings of extraversion (Meredith, 1993; Millsap \& Olivera-Aguilar, 2012) and provide a step-by-step example for conducting LRTs in multiply imputed data. The computer code and the example data can be found in Supplement B and on the OSF project page (https://osf.io/u9s4k). In addition, we 
provide a shorter, annotated version of the code in the Appendix.

In the EIKA study, each personality trait was measured with a set of eight adjective pairs (e.g., "quiet" vs. "talkative") rated on a 5-point Likert scale, some of which were negatively worded and reverse-coded for analysis. The self- and parent ratings had between $19.1 \%$ and $33.5 \%$ missing data. The students' sex, cognitive ability, and reading and math proficiency were missing in $0.3 \%$ to $1.5 \%$ of the cases and were used as auxiliary variables (e.g., Collins et al., 2001). To assess measurement invariance, we compared the following specifications of a twodimensional confirmatory factor analysis (CFA) model: configural (form only, $H_{f}$ ), metric (loadings, $H_{\boldsymbol{\Lambda}}$ ), scalar (loadings and intercepts, $H_{\boldsymbol{\Lambda} \tau}$ ). All models included correlated residuals for the item pairs as well as correlated residuals between the negatively-worded items to account for possible method effects (see also Marsh, 1996). To set the scale of the latent variables, we set the factor means and variances to zero and one, respectively, in the configural model and relaxed them as needed in the metric and scalar invariance models (see Millsap \& Olivera-Aguilar, 2012). All models were estimated with standard maximum likelihood procedures. Given the discrete nature of the item responses, this should be regarded as an approximation (see also DiStefano \& Morgan, 2014; Lei \& Shiverdecker, 2020). We used the R packages mi ce to impute the missing data (van Buuren \& Groothuis-Oudshoorn, 2011), lavaan to fit the analysis models (Rosseel, 2012), and mitml to implement and pool the LRTs with $D_{2}, D_{3}$, and $D_{4}$ (Grund, Robitzsch, et al., 2021). As an alternative, $D_{2}$ and $D_{3}$ can also be implemented with the R package semTools (Jorgensen et al., 2021), which yields the same results (see Supplement B).

The results are summarized in Table 5 and indicated that the data supported metric but not scalar invariance for the self- and parent ratings. On the basis of these findings, we also investigated whether the data supported partial invariance by comparing the metric and scaler invariance models with alternative models that released individual constraints that had been placed on the item parameters (e.g., through modification indices in FIML, or model comparisons in MI). In particular, when we released the constraint on the intercept of Item $3\left(H_{\Lambda \tau(3)}\right)$, these comparisons indicated that scalar invariance held at least partially for all items except Item 3 (see 
Table 57. Consistent with our simulation results, we found that FIML tended to provide larger $\Delta \chi^{2}$ values than MI. However, the results were fairly consistent across the different methods, and all methods led to the same conclusions.

\section{Discussion}

The present article was concerned with pooling methods for LRTs in multiply imputed data sets. In writing this article, we had multiple goals: to provide a review of all methods currently available for pooling LRTs, to evaluate and compare these methods in the context of typical applications in psychology and other fields, and to facilitate their application with a userfriendly implementation in the R package mitml (Grund, Robitzsch, et al., 2021). To this end, we elaborated on the advantages and disadvantages of these methods, evaluated their performance in three simulation studies, and illustrated their use in a step-by-step example that was concerned with testing for measurement invariance in SEM.

Overall, our findings indicate that all methods can provide reliable ways to pool LRTs in multiply imputed data but also that they can behave differently in more challenging situations (e.g., small samples, model comparisons that involve a large number of parameters, large amounts of missing data). Specifically, we found that $D_{2}$ can sometimes be too liberal, whereas $D_{3}$ and $D_{4}$ can be too conservative. The differences between the pooling methods were most pronounced in small samples, with large amounts of missing data, and when the model comparisons involved many parameters. In these situations, we found that $D_{2}$ was usually the most liberal and least reliable among the pooling methods for LRTs, whereas $D_{3}$ and $D_{4}$ usually maintained acceptable Type 1 error rates.

For $D_{2}$ and $D_{3}$, these findings are consistent with the existing literature on the performance of these methods (e.g., Grund et al., 2016; Liu \& Enders, 2017; van Ginkel \& Kroonenberg, 2021). For the novel $D_{4}$ procedure, our findings suggest that the procedure can be considered to be at least as reliable as - if not more reliable than — the $D_{3}$ procedure. This is extremely encouraging because computing $D_{4}$ requires only the construction of a "stacked" data set, which strongly simplifies the application of LRTs with multiply imputed data and should allow for much 
simpler implementations in statistical software that can cover a wider variety of statistical models. The mitml package (Grund, Robitzsch, et al., 2021) implements $D_{4}$-in addition to $D_{2}$ and $D_{3}$ - through the generic logLik () interface, which can be used with many models estimated in R. This includes (generalized) linear models, multilevel models, and SEM, among others.

Missing data are the norm rather than the exception in empirical research. They can be caused by the unintended dropout or nonresponse of study participants as well as planned missing data designs, which have become more and more popular in psychology and related fields (Graham et al., 2006; Rhemtulla et al., 2014; Rhemtulla \& Hancock, 2016). In this article, we focused on MI in its traditional role as a tool for handling missing data. However, the methods considered here also have relevance in other applications. For example, imputed data also occur in large-scale studies, where MI is used to impute latent variable scores (Mislevy, 1991), in causal inference with the potential outcomes framework (Westreich et al., 2015), and in the analysis of synthetic data, where MI is used to overcome disclosure limitations and allow for reproducible analyses with sensitive data (Reiter, 2002; see also Raghunathan, 2021).

\section{Limitations and Future Research}

As in all studies, this study has multiple limitations and suggests different avenues for additional research. First, although we considered a relatively wide variety of statistical models and conditions, it is important to emphasize that our findings need not generalize to other scenarios. For this reason, future research should evaluate these methods in other contexts (e.g., longitudinal designs, multilevel data) and for specific applications of popular model types (e.g., SEM, multilevel models). In the context of SEM, other methods could also be used to conduct LRTs in multiply imputed data (Chung \& Cai, 2019; Lee \& Cai, 2012).

Second, in our simulation studies, we focused on conditions with similar or equal FMIs for the model parameters, which is in line with the assumptions made by the pooling methods for LRTs. Our results from Study 3 and the additional simulation in Supplement A suggested that the procedures were fairly robust against small to moderate violations of this assumption, but more 
research is needed to fully understand the effects of larger differences in the FMIs on the performance of these methods.

Third, although our focus was on model comparisons with LRTs, statistical models are also often compared in terms of goodness-of-fit indices, especially in SEM (e.g., West et al., 2012). Previous research has shown that $D_{3}$ can be used to construct information criteria (Claeskens \& Consentino, 2008) and fit indices in multiply imputed data (Asparouhov \& Muthén, 2008; Enders \& Mansolf, 2018) with properties similar to FIML. However, it has also been shown that computing fit indices for SEM is not straightforward even with FIML because the incompletedata fit function implies different population values for popular fit indices such as the CFI and RMSEA (Lai, 2021; Zhang \& Savalei, 2020). Future research should consider this problem further, including novel methods such as $D_{4}$ and possibly its robust version suggested by Chan and Meng (2022; see also Supplement A).

Finally, model comparisons sometimes require methods other than LRTs. For example, when conducting SEM with categorical or nonnormal data, it has been recommended that researchers use robust or limited-information procedures (e.g., DiStefano \& Morgan, 2014; Lei \& Shiverdecker, 2020) and that statistical models be compared with adjusted or scaled $\chi^{2}$ difference tests (Satorra and Bentler, 2001, 2010; Yuan and Bentler, 2000; see also Shi et al., 2020). Recent research showed that $D_{2}$ can be used in this setting with acceptable results (Liu \& Sriutaisuk, 2020, 2021; Liu et al., 2021). Nonetheless, more research is needed to fully understand the performance of pooling methods for LRTs when applied to categorical or nonnormal data and how each method can be adapted to address scaled $\chi^{2}$ difference tests in nonnormal SEM. Furthermore, when there are missing values in nonnormal or categorical data, choosing an impuation method is sometimes not straightforward (Quartagno and Carpenter, 2019; see also Du et al., 2022; Hughes et al., 2014). Because this choice could also affect the performances of the pooling methods for LRTs, future studies should also consider the interplay between imputation and pooling methods in applications with nonnormal or categorical data.

In sum, we hope that the present article provides a first step toward a more complete 
understanding of pooling methods for LRTs and similar procedures for model comparisons in multiply imputed data. 


\section{References}

Agresti, A. (2007). An introduction to categorical data analysis (2nd ed.). Wiley.

Arbuckle, J. L. (1996). Full information estimation in the presence of incomplete data. In G. A. Marcoulides \& R. E. Schumacker (Eds.), Advanced structural equation modeling: Issues and techniques. Erlbaum.

Asparouhov, T., \& Muthén, B. O. (2008). Chi-square statistics with multiple imputation (Technical Appendix). http://www.statmodel.com/

Barnard, J., \& Rubin, D. B. (1999). Small-sample degrees of freedom with multiple imputation. Biometrika, 86, 948-955. https://doi.org/10.1093/biomet/86.4.948

Bodner, T. E. (2008). What improves with increased missing data imputations? Structural Equation Modeling: A Multidisciplinary Journal, 15, 651-675. https://doi.org/10.1080/10705510802339072

Bradley, J. V. (1978). Robustness? British Journal of Mathematical and Statistical Psychology, 31, 144-152. https://doi.org/10.1111/j.2044-8317.1978.tb00581.x

Buse, A. (1982). The likelihood ratio, Wald, and Lagrange multiplier tests: An expository note. The American Statistician, 36, 153-157.

Chan, K. W., \& Meng, X.-L. (2019). Multiple improvements of multiple imputation likelihood ratio tests. https://arxiv.org/abs/1711.08822

Chan, K. W., \& Meng, X.-L. (2022). Multiple improvements of multiple imputation likelihood ratio tests. Statistica Sinica. https://doi.org/10.5705/ss.202019.0314

Chung, S., \& Cai, L. (2019). Alternative multiple imputation inference for categorical structural equation modeling. Multivariate Behavioral Research, 54, 323-337. https://doi.org/10.1080/00273171.2018.1523000

Claeskens, G., \& Consentino, F. (2008). Variable selection with incomplete covariate data. Biometrics, 64, 1062-1069. https://doi.org/10.1111/j.1541-0420.2008.01003.x

Collins, L. M., Schafer, J. L., \& Kam, C.-M. (2001). A comparison of inclusive and restrictive strategies in modern missing data procedures. Psychological Methods, 6, 330-351. 
https://doi.org/10.1037/1082-989X.6.4.330

Cox, D. R. (2006). Principles of statistical inference. Cambridge University Press.

DiStefano, C., \& Morgan, G. B. (2014). A comparison of diagonal weighted least squares robust estimation techniques for ordinal data. Structural Equation Modeling: A Multidisciplinary Journal, 21, 425-438. https://doi.org/10.1080/10705511.2014.915373

Du, H., Alacam, E., Mena, S., \& Keller, B. T. (2022). Compatibility in imputation specification. Behavior Research Methods. https://doi.org/10.3758/s13428-021-01749-5

EIKA. (2006). Projekt Entwicklung und Implementierung eines neuen Konzeptes zur Eingliederung Jugendlicher in die Berufs- und Arbeitswelt in Schulen mit erhöhtem Förderbedarf (Unpublished data set). Humboldt-Universität zu Berlin, FriedrichAlexander-Universität Erlangen Nürnberg. Berlin, Erlangen-Nürnberg.

Enders, C. K. (2010). Applied missing data analysis. Guilford Press.

Enders, C. K., \& Mansolf, M. (2018). Assessing the fit of structural equation models with multiply imputed data. Psychological Methods, 23, 76-93. https://doi.org/10.1037/met0000102

Graham, J. W., Olchowski, A. E., \& Gilreath, T. D. (2007). How many imputations are really needed? Some practical clarifications of multiple imputation theory. Prevention Science, 8, 206-213. https://doi.org/10.1007/s11121-007-0070-9

Graham, J. W., Taylor, B. J., Olchowski, A. E., \& Cumsille, P. E. (2006). Planned missing data designs in psychological research. Psychological Methods, 11, 323-343. https://doi.org/10.1037/1082-989X.11.4.323

Grund, S., Lüdtke, O., \& Robitzsch, A. (2016). Pooling ANOVA results from multiply imputed datasets: A simulation study. Methodology, 12, 75-88. https://doi.org/10.1027/1614-2241/a000111

Grund, S., Lüdtke, O., \& Robitzsch, A. (2021). Pooling methods for likelihood ratio tests in multiply imputed data sets. Open Science Framework. https://doi.org/10.17605/osf.io/u9s4k

Grund, S., Robitzsch, A., \& Luedtke, O. (2021). mitml: Tools for multiple imputation in multilevel 
modeling (Version 0.4-1). https://CRAN.R-project.org/package=mitml

Held, L., \& Bové, D. S. (2014). Applied Statistical Inference: Likelihood and Bayes. Springer. https://doi.org/10.1007/978-3-642-37887-4

Hughes, R. A., White, I. R., Seaman, S. R., Carpenter, J. R., Tilling, K., \& Sterne, J. A. (2014). Joint modelling rationale for chained equations. BMC Medical Research Methodology, 14(28), 1-10. https://doi.org/10.1186/1471-2288-14-28

Jöreskog, K. G. (1979). Statistical models and methods for analysis of longitudinal data. In K. G. Jöreskog, D. Sörbom, \& J. Magidson (Eds.), Advances in factor analysis and structural equation models (pp. 129-170). Abt books.

Jorgensen, T. D., Pornprasertmanit, S., Schoemann, A. M., Rosseel, Y., Miller, P., Quick, C., Garnier-Villarreal, M., Selig, J., Boulton, A., Preacher, K., Coffman, D., Rhemtulla, M., Robitzsch, A., Enders, C., Arslan, R., Clinton, B., Panko, P., Merkle, E., Chesnut, S., .. . Johnson, A. R. (2021). semTools: Useful tools for structural equation modeling (Version 0.5-5). https://CRAN.R-project.org/package=semTools

Lai, K. (2021). Correct estimation methods for RMSEA under missing data. Structural Equation Modeling: A Multidisciplinary Journal, 28, 207-2018. https://doi.org/10.1080/10705511.2020.1755864

Lang, K. M., \& Little, T. D. (2014). The supermatrix technique: A simple framework for hypothesis testing with missing data. International Journal of Behavioral Development, 38(5), 461-470. https://doi.org/10.1177/0165025413514326

Lee, T., \& Cai, L. (2012). Alternative multiple imputation inference for mean and covariance structure modeling. Journal of Educational and Behavioral Statistics, 37, 675-702. https://doi.org/10.3102/1076998612458320

Lei, P.-W., \& Shiverdecker, L. K. (2020). Performance of estimators for confirmatory factor analysis of ordinal variables with missing data. Structural Equation Modeling: A Multidisciplinary Journal, 27, 584-601. https://doi.org/10.1080/10705511.2019.1680292

Li, K. H., Meng, X.-L., Raghunathan, T. E., \& Rubin, D. B. (1991). Significance levels from 
repeated $p$-values with multiply-imputed data. Statistica Sinica, 1, 65-92. http://www.stat.sinica.edu.tw/statistica/

Li, K. H., Raghunathan, T. E., \& Rubin, D. B. (1991). Large-sample significance levels from multiply imputed data using moment-based statistics and an $F$ reference distribution. Journal of the American Statistical Association, 86, 1065-1073. https://doi.org/10.1080/01621459.1991.10475152

Little, T. D. (2013). Longitudinal structural equation modeling. The Guilford Press.

Liu, Y., \& Enders, C. K. (2017). Evaluation of multi-parameter test statistics for multiple imputation. Multivariate Behavioral Research, 52, 371-390. https://doi.org/10.1080/00273171.2017.1298432

Liu, Y., \& Sriutaisuk, S. (2020). Evaluation of model fit in structural equation models with ordinal missing data: An examination of the $D_{2}$ method. Structural Equation Modeling: $A$ Multidisciplinary Journal, 27, 561-583. https://doi.org/10.1080/10705511.2019.1662307

Liu, Y., \& Sriutaisuk, S. (2021). A comparison of FIML- versus multiple-imputation-based methods to test measurement invariance with incomplete ordinal variables. Structural Equation Modeling: A Multidisciplinary Journal, 28, 590-608. https://doi.org/10.1080/10705511.2021.1876520

Liu, Y., Sriutaisuk, S., \& Chung, S. (2021). Evaluation of model fit in structural equation models with ordinal missing data: A comparison of the $D_{2}$ and MI2S methods. Structural Equation Modeling: A Multidisciplinary Journal. Advance online publication. https://doi.org/10.1080/10705511.2021.1919118

Mansolf, M., Jorgensen, T. D., \& Enders, C. K. (2020). A multiple imputation score test for model modification in structural equation models. Psychological Methods, 25, 393-411. https://doi.org/10.1037/met0000243

Marsh, H. W. (1989). Confirmatory factor analyses of multitrait-multimethod data: Many problems and a few solutions. Applied Psychological Measurement, 13, 335-361. https://doi.org/10.1177/014662168901300402 
Marsh, H. W. (1996). Positive and negative global self-esteem: A substantively meaningful distinction or artifactors? Journal of Personality and Social Psychology, 70, 810-819. https://doi.org/10.1037/0022-3514.70.4.810

Medeiros, R. (2008). Likelihood ratio tests for multiply imputed datasets: Introducing milrtest (Presentation). Fall North American Stata Users Group Meeting, San Francisco, CA, United States. https://www.stata.com/meeting/proceedings/

Meng, X.-L., \& Rubin, D. B. (1992). Performing likelihood ratio tests with multiply-imputed data sets. Biometrika, 79, 103-111. https://doi.org/10.1093/biomet/79.1.103

Meredith, W. (1993). Measurement invariance, factor analysis and factorial invariance. Psychometrika, 58, 525-543. https://doi.org/10.1007/BF02294825

Millsap, R. E., \& Olivera-Aguilar, M. (2012). Investigating measurement invariance using confirmatory factor analysis. Handbook of structural equation modeling. Guilford Press.

Mislevy, R. J. (1991). Randomization-based inference about latent variables from complex samples. Psychometrika, 56, 177-196. https://doi.org/10.1007/BF02294457

Mistler, S. A. (2013). A SAS macro for computing pooled likelihood ratio tests with multiply imputed data. Proceedings of the SAS Global Forum. http://support.sas.com/

Muthén, L. K., \& Muthén, B. O. (2012). Mplus user’s guide (7th ed.). Muthén \& Muthén.

Quartagno, M., \& Carpenter, J. R. (2019). Multiple imputation for discrete data: Evaluation of the joint latent normal model. Biometrical Journal, 61, 1003-1019. https://doi.org/10.1002/bimj.201800222

R Core Team. (2021). R: A language and environment for statistical computing (Version 4.1). https://www.R-project.org/

Raghunathan, T. E. (2021). Synthetic data. Annual Review of Statistics and Its Application, 8, 129-140. https://doi.org/10.1146/annurev-statistics-040720-031848

Reiter, J. P. (2007). Small-sample degrees of freedom for multi-component significance tests with multiple imputation for missing data. Biometrika, 94, 502-508. https://doi.org/10.1093/biomet/asm028 
Reiter, J. P. (2002). Satisfying disclosure restrictions with synthetic data sets. Journal of Official Statistics, 18, 531-543.

Reiter, J. P., \& Raghunathan, T. E. (2007). The multiple adaptations of multiple imputation. Journal of the American Statistical Association, 102, 1462-1471. https://doi.org/10.1198/016214507000000932

Rhemtulla, M., Jia, F., Wu, W., \& Little, T. D. (2014). Planned missing designs to optimize the efficiency of latent growth parameter estimates. International Journal of Behavioral Development, 38, 423-434. https://doi.org/10.1177/0165025413514324

Rhemtulla, M., \& Hancock, G. R. (2016). Planned missing data designs in educational psychology research. Educational Psychologist, 51, 305-316. https://doi.org/10.1080/00461520.2016.1208094

Robitzsch, A., Grund, S., \& Henke, T. (2021). miceadds: Some additional multiple imputation functions, especially for mice (Version 3.11-6). https://CRAN.R-project.org/package=miceadds

Rosseel, Y. (2012). lavaan: An R package for structural equation modeling. Journal of Statistical Software, 48(2), 1-36. https://doi.org/10.18637/jss.v048.i02

Rubin, D. B. (1987). Multiple imputation for nonresponse in surveys. Wiley.

Satorra, A., \& Bentler, P. M. (2001). A scaled difference chi-square test statistic for moment structure analysis. Psychometrika, 66, 507-514. https://doi.org/10.1007/BF02296192

Satorra, A., \& Bentler, P. M. (2010). Ensuring positiveness of the scaled difference chi-square test statistic. Psychometrika, 75, 243-248. https://doi.org/10.1007/s11336-009-9135-y

Savalei, V., \& Rhemtulla, M. (2012). On obtaining estimates of the fraction of missing information from full information maximum likelihood. Structural Equation Modeling: A Multidisciplinary Journal, 19, 477-494. https://doi.org/10.1080/10705511.2012.687669

Schafer, J. L. (1997). Analysis of incomplete multivariate data. CRC Press.

Schafer, J. L., \& Graham, J. W. (2002). Missing data: Our view of the state of the art. Psychological Methods, 7, 147-177. https://doi.org/10.1037//1082-989X.7.2.147 
Shi, D., Maydeu-Olivares, A., \& Rosseel, Y. (2020). Assessing fit in ordinal factor analysis models: SRMR vs. RMSEA. Structural Equation Modeling: A Multidisciplinary Journal, 27, 1-15. https://doi.org/10.1080/10705511.2019.1611434

van Buuren, S. (2018). Flexible imputation of missing data (2nd ed.). CRC Press. https://stefvanbuuren.name/fimd/

van Buuren, S., Brand, J. P. L., Groothuis-Oudshoorn, C. G. M., \& Rubin, D. B. (2006). Fully conditional specification in multivariate imputation. Journal of Statistical Computation and Simulation, 76, 1049-1064. https://doi.org/10.1080/10629360600810434

van Buuren, S., \& Groothuis-Oudshoorn, K. (2011). mice: Multivariate imputation by chained equations in R. Journal of Statistical Software, 45(3), 1-67. https://doi.org/10.18637/jss.v045.i03

van Ginkel, J. R., \& Kroonenberg, P. M. (2021). Multiple imputation to balance unbalanced designs for two-way analysis of variance. Methodology, 17(1), 39-57. https://doi.org/10.5964/meth.6085

von Hippel, P. T. (2020). How many imputations do you need? A two-stage calculation using a quadratic rule. Sociological Methods \& Research, 49, 699-718. https://doi.org/10.1177/0049124117747303

West, S. G., Taylor, A. B., \& Wu, W. (2012). Model fit and model selection in structural equation modeling. In R. H. Hoyle (Ed.), Handbook of structural equation modeling. Guilford Press.

Westreich, D., Edwards, J. K., Cole, S. R., Platt, R. W., Mumford, S. L., \& Schisterman, E. F. (2015). Imputation approaches for potential outcomes in causal inference. International Journal of Epidemiology, 44, 1731-1737. https://doi.org/10.1093/ije/dyv135

Wood, A. M., White, I. R., \& Royston, P. (2008). How should variable selection be performed with multiply imputed data? Statistics in Medicine, 27, 3227-3246. https://doi.org/10.1002/sim.3177

Yuan, K.-H., \& Bentler, P. M. (2000). Three likelihood-based methods for mean and covariance structure analysis with nonnormal missing data. Sociological Methodology, 30, 165-200. 
https://doi.org/10.1111/0081-1750.00078

Zhang, X., \& Savalei, V. (2020). Examining the effect of missing data on RMSEA and CFI under normal theory full-information maximum likelihood. Structural Equation Modeling: A Multidisciplinary Journal, 27, 219-239. https://doi.org/10.1080/10705511.2019.1642111 
Table 1

Study 1: Type 1 Error Rates in $\%\left(R^{2}=0\right)$ in Conditions with MAR Data

\begin{tabular}{|c|c|c|c|c|c|c|c|c|c|}
\hline \multirow[b]{2}{*}{$\mathrm{MD}$} & \multirow[b]{2}{*}{$k$} & \multirow[b]{2}{*}{$n$} & \multirow[b]{2}{*}{ FMI } & \multirow[b]{2}{*}{$\mathrm{CD}$} & \multirow[b]{2}{*}{ LD } & \multirow[b]{2}{*}{ FIML } & \multicolumn{3}{|c|}{ MI } \\
\hline & & & & & & & $D_{2}$ & $D_{3}$ & $D_{4}$ \\
\hline \multirow[t]{12}{*}{$10 \%$} & \multirow[t]{4}{*}{2} & 50 & .194 & 6.3 & 5.5 & 5.7 & 6.2 & 5.3 & 5.4 \\
\hline & & 100 & .194 & 6.7 & 5.9 & 6.3 & 6.8 & 5.9 & 5.9 \\
\hline & & 200 & .248 & 5.4 & 4.9 & 5.3 & 5.3 & 5.5 & 5.5 \\
\hline & & 500 & .200 & 5.9 & 6.3 & 5.6 & 5.6 & 5.3 & 5.3 \\
\hline & \multirow[t]{4}{*}{4} & 50 & .176 & 6.9 & 6.8 & 6.6 & 7.5 & 5.3 & 5.5 \\
\hline & & 100 & .230 & 6.1 & 6.7 & 6.7 & 6.9 & 6.4 & 6.5 \\
\hline & & 200 & .213 & 5.3 & 5.2 & 4.9 & 4.9 & 4.7 & 4.7 \\
\hline & & 500 & .221 & 5.3 & 5.8 & 4.5 & 4.6 & 4.5 & 4.5 \\
\hline & \multirow[t]{4}{*}{6} & 50 & .154 & 7.7 & 9.1 & 7.9 & 9.1 & 5.6 & 5.9 \\
\hline & & 100 & .200 & 6.2 & 7.5 & 7.2 & 8.1 & 6.0 & 6.3 \\
\hline & & 200 & .217 & 6.7 & 7.6 & 6.9 & 7.4 & 7.0 & 7.1 \\
\hline & & 500 & .230 & 5.2 & 4.9 & 4.6 & 5.0 & 4.3 & 4.3 \\
\hline \multirow[t]{12}{*}{$20 \%$} & \multirow[t]{4}{*}{2} & 50 & .307 & 6.6 & 6.5 & 6.8 & 7.3 & 5.4 & 5.6 \\
\hline & & 100 & .346 & 6.0 & 6.5 & 6.3 & 6.6 & 5.9 & 5.9 \\
\hline & & 200 & .358 & 6.1 & 5.8 & 6.0 & 6.3 & 6.0 & 6.0 \\
\hline & & 500 & .370 & 4.5 & 4.8 & 4.6 & 4.9 & 4.6 & 4.6 \\
\hline & \multirow[t]{4}{*}{4} & 50 & .260 & 7.5 & 7.1 & 6.7 & 8.2 & 3.7 & 4.1 \\
\hline & & 100 & .335 & 7.1 & 6.7 & 6.8 & 7.9 & 5.3 & 5.5 \\
\hline & & 200 & .356 & 5.9 & 5.6 & 5.8 & 6.9 & 5.3 & 5.3 \\
\hline & & 500 & .364 & 5.0 & 5.0 & 4.4 & 5.3 & 4.0 & 4.0 \\
\hline & \multirow[t]{4}{*}{6} & 50 & .250 & 7.8 & 9.7 & 8.8 & 12.4 & 3.5 & 4.1 \\
\hline & & 100 & .293 & 7.2 & 7.2 & 7.3 & 9.6 & 5.1 & 5.5 \\
\hline & & 200 & .354 & 5.7 & 6.0 & 6.0 & 7.1 & 4.9 & 5.1 \\
\hline & & 500 & .385 & 4.9 & 5.1 & 4.3 & 5.3 & 4.3 & 4.4 \\
\hline \multirow[t]{12}{*}{$30 \%$} & \multirow[t]{4}{*}{2} & 50 & .418 & 5.5 & 5.9 & 5.6 & 6.2 & 2.9 & 3.1 \\
\hline & & 100 & .472 & 4.7 & 5.8 & 5.5 & 6.3 & 4.5 & 4.7 \\
\hline & & 200 & .476 & 4.9 & 5.3 & 5.0 & 5.3 & 4.1 & 4.2 \\
\hline & & 500 & .512 & 5.5 & 4.3 & 5.5 & 5.5 & 5.3 & 5.3 \\
\hline & \multirow[t]{4}{*}{4} & 50 & .381 & 6.6 & 9.2 & 7.9 & 11.1 & 2.5 & 3.2 \\
\hline & & 100 & .440 & 5.4 & 6.6 & 6.5 & 8.9 & 3.8 & 4.3 \\
\hline & & 200 & .453 & 6.3 & 5.9 & 5.6 & 6.7 & 4.9 & 5.3 \\
\hline & & 500 & .483 & 4.8 & 4.8 & 4.9 & 5.6 & 4.6 & 4.7 \\
\hline & \multirow[t]{4}{*}{6} & 50 & .339 & 7.8 & 11.0 & 10.3 & 16.5 & 1.2 & 1.9 \\
\hline & & 100 & .413 & 6.3 & 7.5 & 6.9 & 10.7 & 3.6 & 4.2 \\
\hline & & 200 & .465 & 5.7 & 6.0 & 6.2 & 9.6 & 4.6 & 5.0 \\
\hline & & 500 & .481 & 5.2 & 5.8 & 5.3 & 7.9 & 5.1 & 5.3 \\
\hline
\end{tabular}

Note. Bold numbers refer to Type 1 error rates outside the $[2.5 \% ; 7.5 \%]$ interval. $k=$ number of predictors; $n=$ sample size $; \mathrm{FMI}=$ empirical fraction of missing information per condition; $\mathrm{CD}=$ complete data $\mathrm{LD}=$ listwise deletion; FIML = full-information maximum likelihood; $\mathrm{MI}=$ multiple imputation; $D_{2}, D_{3}, D_{4}=$ pooling methods. 
Table 2

Study 2: Type 1 Error Rates in \% $\left(R^{2}=0\right)$ in Conditions with MAR Data

\begin{tabular}{|c|c|c|c|c|c|c|c|c|c|}
\hline \multirow[b]{2}{*}{ MD } & \multirow[b]{2}{*}{$k$} & \multirow[b]{2}{*}{$n$} & \multirow[b]{2}{*}{ FMI } & \multirow[b]{2}{*}{$\mathrm{CD}$} & \multirow[b]{2}{*}{$\mathrm{LD}$} & \multirow[b]{2}{*}{ FIML } & \multicolumn{3}{|c|}{ MI } \\
\hline & & & & & & & $D_{2}$ & $D_{3}$ & $D_{4}$ \\
\hline \multirow{12}{*}{$10 \%$} & \multirow[t]{4}{*}{2} & 100 & .138 & 5.7 & 5.6 & 5.5 & 5.2 & 5.0 & 5.0 \\
\hline & & 200 & .153 & 5.5 & 5.5 & 5.1 & 5.5 & 5.5 & 5.5 \\
\hline & & 500 & .135 & 4.3 & 4.9 & 4.9 & 4.9 & 4.9 & 4.9 \\
\hline & & 1000 & .163 & 5.5 & 5.4 & 7.0 & 6.5 & 7.1 & 7.1 \\
\hline & \multirow[t]{4}{*}{4} & 100 & .147 & 6.2 & 6.4 & 5.5 & 5.7 & 5.0 & 5.0 \\
\hline & & 200 & .149 & 4.8 & 5.0 & 4.8 & 4.3 & 4.3 & 4.3 \\
\hline & & 500 & .120 & 5.3 & 4.2 & 4.1 & 4.3 & 4.2 & 4.2 \\
\hline & & 1000 & .138 & 4.6 & 4.4 & 4.5 & 4.6 & 4.6 & 4.6 \\
\hline & \multirow[t]{4}{*}{6} & 100 & .154 & 6.5 & 6.9 & 6.3 & 6.2 & 5.3 & 5.3 \\
\hline & & 200 & .144 & 5.9 & 5.5 & 5.9 & 5.9 & 5.3 & 5.3 \\
\hline & & 500 & .142 & 6.5 & 6.4 & 6.9 & 6.9 & 6.8 & 6.8 \\
\hline & & 1000 & .140 & 3.7 & 3.7 & 4.1 & 4.1 & 4.1 & 4.1 \\
\hline \multirow[t]{12}{*}{$20 \%$} & \multirow[t]{4}{*}{2} & 100 & .335 & 5.5 & 5.3 & 5.4 & 5.8 & 4.8 & 4.9 \\
\hline & & 200 & .323 & 4.6 & 4.9 & 5.7 & 5.4 & 4.7 & 4.8 \\
\hline & & 500 & .293 & 5.9 & 5.3 & 5.1 & 5.1 & 4.9 & 4.9 \\
\hline & & 1000 & .288 & 4.7 & 4.5 & 4.8 & 4.4 & 4.7 & 4.7 \\
\hline & \multirow[t]{4}{*}{4} & 100 & .334 & 6.2 & 5.7 & 5.6 & 5.6 & 3.9 & 4.2 \\
\hline & & 200 & .329 & 5.3 & 5.8 & 5.8 & 5.6 & 5.1 & 5.2 \\
\hline & & 500 & .302 & 6.0 & 5.2 & 4.9 & 5.8 & 4.9 & 5.0 \\
\hline & & 1000 & .309 & 4.2 & 5.3 & 4.4 & 5.1 & 4.3 & 4.4 \\
\hline & \multirow[t]{4}{*}{6} & 100 & .361 & 6.0 & 7.3 & 6.2 & 7.2 & 4.1 & 4.4 \\
\hline & & 200 & .332 & 5.1 & 5.9 & 4.8 & 5.6 & 4.1 & 4.2 \\
\hline & & 500 & .311 & 5.1 & 5.9 & 5.3 & 5.7 & 5.2 & 5.2 \\
\hline & & 1000 & .278 & 4.7 & 5.1 & 4.4 & 4.4 & 4.1 & 4.1 \\
\hline \multirow[t]{12}{*}{$30 \%$} & \multirow[t]{4}{*}{2} & 100 & .519 & 5.9 & 5.9 & 5.5 & 6.1 & 4.4 & 4.9 \\
\hline & & 200 & .485 & 5.8 & 5.1 & 5.5 & 6.0 & 5.4 & 5.5 \\
\hline & & 500 & .491 & 4.8 & 6.2 & 5.7 & 5.7 & 5.1 & 5.2 \\
\hline & & 1000 & .485 & 5.8 & 3.9 & 6.2 & 6.5 & 6.4 & 6.4 \\
\hline & \multirow[t]{4}{*}{4} & 100 & .544 & 6.4 & 7.8 & 6.7 & 8.0 & 3.9 & 4.2 \\
\hline & & 200 & .502 & 5.2 & 6.0 & 5.4 & 6.4 & 4.3 & 4.5 \\
\hline & & 500 & .465 & 5.2 & 5.3 & 5.5 & 6.1 & 5.3 & 5.4 \\
\hline & & 1000 & .474 & 6.2 & 5.5 & 4.0 & 5.5 & 5.0 & 5.0 \\
\hline & \multirow[t]{4}{*}{6} & 100 & .553 & 6.3 & 7.5 & 6.7 & 8.6 & 2.8 & 3.6 \\
\hline & & 200 & .497 & 5.9 & 6.3 & 5.5 & 6.7 & 4.2 & 4.7 \\
\hline & & 500 & .490 & 5.2 & 5.5 & 5.5 & 6.6 & 5.3 & 5.4 \\
\hline & & 1000 & .469 & 4.5 & 6.1 & 4.7 & 6.2 & 5.2 & 5.3 \\
\hline
\end{tabular}

Note. Bold numbers refer to Type 1 error rates outside the $[2.5 \% ; 7.5 \%]$ interval. $k=$ number of predictors; $n=$ sample size $; \mathrm{FMI}=$ empirical fraction of missing information per condition; $\mathrm{CD}=$ complete data; $\mathrm{LD}=$ listwise deletion; FIML = full-information maximum likelihood; $\mathrm{MI}=$ multiple imputation; $D_{2}, D_{3}, D_{4}=$ pooling methods. 
Table 3

Specification of Factor Loadings $\boldsymbol{\Lambda}=\left(\boldsymbol{\lambda}_{1}, \boldsymbol{\lambda}_{2}\right)$ in Study 3

\begin{tabular}{|c|c|c|c|c|c|c|}
\hline & \multicolumn{2}{|c|}{$k=4$} & \multicolumn{2}{|c|}{$k=6$} & \multicolumn{2}{|c|}{$k=8$} \\
\hline & $\lambda_{1}$ & $\lambda_{2}$ & $\lambda_{1}$ & $\lambda_{2}$ & $\lambda_{1}$ & $\lambda_{2}$ \\
\hline$y_{11}$ & 0.70 & 0 & 0.70 & 0 & 0.75 & 0 \\
\hline$y_{12}$ & 0.78 & 0 & 0.75 & 0 & 0.78 & 0 \\
\hline$y_{13}$ & 0.87 & 0 & 0.80 & 0 & 0.81 & 0 \\
\hline$y_{14}$ & 0.95 & 0 & 0.85 & 0 & 0.84 & 0 \\
\hline$y_{15}$ & & & 0.90 & 0 & 0.86 & 0 \\
\hline$y_{16}$ & & & 0.95 & 0 & 0.89 & 0 \\
\hline$y_{17}$ & & & & & 0.92 & 0 \\
\hline$y_{18}$ & & & & & 0.95 & 0 \\
\hline$y_{21}$ & 0 & 0.70 & 0 & 0.70 & 0 & 0.75 \\
\hline$y_{22}$ & 0 & 0.78 & 0 & 0.75 & 0 & 0.78 \\
\hline$y_{23}$ & 0 & 0.87 & 0 & 0.80 & 0 & 0.81 \\
\hline$y_{24}$ & 0 & 0.95 & 0 & 0.85 & 0 & 0.84 \\
\hline$y_{25}$ & & & 0 & 0.90 & 0 & 0.86 \\
\hline$y_{26}$ & & & 0 & 0.95 & 0 & 0.89 \\
\hline$y_{27}$ & & & & & 0 & 0.92 \\
\hline$y_{28}$ & & & & & 0 & 0.95 \\
\hline
\end{tabular}

Note. $k=$ number of items per factor. 
Table 4

Study 3: Type 1 Error Rates in \% $(\rho=0)$ in Conditions with MAR Data

\begin{tabular}{|c|c|c|c|c|c|c|c|c|c|}
\hline \multirow[b]{2}{*}{ MD } & \multirow[b]{2}{*}{$k$} & \multirow[b]{2}{*}{$n$} & \multirow[b]{2}{*}{ FMI } & \multirow[b]{2}{*}{$\mathrm{CD}$} & \multirow[b]{2}{*}{ LD } & \multirow[b]{2}{*}{ FIML } & \multicolumn{3}{|c|}{ MI } \\
\hline & & & & & & & $D_{2}$ & $D_{3}$ & $D_{4}$ \\
\hline \multirow[t]{12}{*}{$10 \%$} & \multirow[t]{4}{*}{4} & 100 & .153 & 6.0 & 6.4 & 6.3 & 6.1 & 5.3 & 5.3 \\
\hline & & 200 & .129 & 5.1 & 5.5 & 5.6 & 5.5 & 5.1 & 5.1 \\
\hline & & 500 & .132 & 4.7 & 5.9 & 5.4 & 5.4 & 5.3 & 5.3 \\
\hline & & 1000 & .118 & 5.5 & 4.4 & 4.7 & 4.9 & 4.6 & 4.6 \\
\hline & \multirow[t]{4}{*}{6} & 100 & .135 & 6.3 & 6.8 & 6.8 & 5.8 & 4.8 & 4.7 \\
\hline & & 200 & .132 & 4.9 & 5.2 & 5.1 & 4.9 & 4.5 & 4.5 \\
\hline & & 500 & .121 & 5.9 & 6.5 & 6.1 & 6.2 & 6.0 & 6.0 \\
\hline & & 1000 & .122 & 4.7 & 5.6 & 5.4 & 4.9 & 4.8 & 4.8 \\
\hline & \multirow[t]{4}{*}{8} & 100 & .129 & 6.9 & 7.2 & 6.9 & 5.5 & 5.0 & 4.9 \\
\hline & & 200 & .126 & 5.9 & 5.9 & 6.0 & 5.4 & 5.0 & 4.9 \\
\hline & & 500 & .114 & 5.8 & 5.3 & 4.9 & 4.8 & 4.6 & 4.6 \\
\hline & & 1000 & .110 & 4.9 & 4.1 & 4.8 & 4.4 & 4.3 & 4.3 \\
\hline \multirow[t]{12}{*}{$20 \%$} & \multirow[t]{4}{*}{4} & 100 & .292 & 5.6 & 7.0 & 6.6 & 6.4 & 4.8 & 4.5 \\
\hline & & 200 & .257 & 6.2 & 6.8 & 6.1 & 5.9 & 5.0 & 4.8 \\
\hline & & 500 & .235 & 5.5 & 5.3 & 5.2 & 5.0 & 4.8 & 4.7 \\
\hline & & 1000 & .256 & 4.7 & 6.1 & 5.9 & 6.0 & 5.6 & 5.5 \\
\hline & \multirow[t]{4}{*}{6} & 100 & .272 & 6.9 & 7.0 & 6.4 & 6.1 & 4.2 & 3.9 \\
\hline & & 200 & .250 & 6.0 & 5.8 & 5.3 & 4.9 & 4.0 & 3.8 \\
\hline & & 500 & .236 & 3.9 & 4.7 & 4.6 & 4.5 & 3.8 & 3.8 \\
\hline & & 1000 & .233 & 5.8 & 6.1 & 6.4 & 6.2 & 5.5 & 5.5 \\
\hline & \multirow[t]{4}{*}{8} & 100 & .279 & 6.8 & 8.3 & 7.6 & 5.5 & 3.5 & 3.0 \\
\hline & & 200 & .241 & 5.2 & 6.2 & 5.5 & 4.9 & 3.9 & 3.9 \\
\hline & & 500 & .231 & 5.3 & 4.6 & 4.9 & 4.9 & 4.5 & 4.3 \\
\hline & & 1000 & .222 & 5.0 & 5.7 & 5.9 & 6.1 & 5.6 & 5.6 \\
\hline \multirow[t]{12}{*}{$30 \%$} & \multirow[t]{4}{*}{4} & 100 & .413 & 6.6 & 7.7 & 7.0 & 6.5 & 3.5 & 2.9 \\
\hline & & 200 & .383 & 5.9 & 6.8 & 6.3 & 6.5 & 4.9 & 4.8 \\
\hline & & 500 & .364 & 5.1 & 5.9 & 5.4 & 5.5 & 4.4 & 4.3 \\
\hline & & 1000 & .368 & 5.3 & 5.2 & 5.1 & 5.4 & 4.7 & 4.6 \\
\hline & \multirow[t]{4}{*}{6} & 100 & .407 & 6.1 & 8.9 & 7.7 & 5.8 & 3.0 & 2.4 \\
\hline & & 200 & .366 & 5.3 & 6.7 & 5.6 & 6.0 & 3.5 & 3.4 \\
\hline & & 500 & .347 & 5.1 & 5.4 & 5.1 & 5.4 & 4.3 & 4.1 \\
\hline & & 1000 & .333 & 4.5 & 5.2 & 4.6 & 5.5 & 4.1 & 4.1 \\
\hline & \multirow[t]{4}{*}{8} & 100 & .406 & 6.0 & 8.2 & 7.3 & 4.4 & 1.6 & 1.3 \\
\hline & & 200 & .373 & 5.1 & 6.2 & 6.3 & 5.7 & 3.5 & 3.3 \\
\hline & & 500 & .318 & 5.6 & 5.3 & 4.5 & 4.0 & 3.1 & 3.0 \\
\hline & & 1000 & .332 & 4.7 & 4.9 & 4.8 & 5.2 & 4.0 & 4.0 \\
\hline
\end{tabular}

Note. Bold numbers refer to Type 1 error rates outside the $[2.5 \% ; 7.5 \%]$ interval. $k=$ number of predictors; $n=$ sample size; FMI = empirical fraction of missing information per condition; $\mathrm{CD}=$ complete data; $\mathrm{LD}=$ listwise deletion; FIML = full-information maximum likelihood; $\mathrm{MI}=$ multiple imputation; $D_{2}, D_{3}, D_{4}=$ pooling methods. 
Table 5

Results for the Assessment of Measurement Invariance for Self-and Parent Ratings of Students' Extraversion in the Example Analyses

\begin{tabular}{|c|c|c|c|c|c|c|c|c|c|c|}
\hline \multirow[b]{2}{*}{ Model } & \multirow[b]{2}{*}{ Comparison } & \multirow[b]{2}{*}{$\Delta \mathrm{df}$} & \multicolumn{2}{|c|}{ FIML } & \multicolumn{2}{|c|}{$D_{2}$} & \multicolumn{2}{|c|}{$D_{3}$} & \multicolumn{2}{|c|}{$D_{4}$} \\
\hline & & & $\Delta \chi^{2}$ & $p$ & $\Delta \chi^{2}$ & $p$ & $\Delta \chi^{2}$ & $p$ & $\Delta \chi^{2}$ & $p$ \\
\hline Metric & $H_{f}$ vs. $H_{\boldsymbol{\Lambda}}$ & 7 & 7.8 & .351 & 6.5 & .480 & 7.3 & .397 & 7.3 & .396 \\
\hline Scalar & $H_{\boldsymbol{\Lambda}}$ vs. $H_{\boldsymbol{\Lambda} \boldsymbol{\tau}}$ & 7 & 28.0 & .000 & 27.3 & .000 & 27.1 & .000 & 27.1 & .000 \\
\hline Partial scalar & $H_{\boldsymbol{\Lambda}}$ vs. $H_{\boldsymbol{\Lambda} \boldsymbol{\tau}(3)}$ & 6 & 6.6 & .355 & 7.0 & .318 & 6.6 & .357 & 6.6 & .357 \\
\hline
\end{tabular}

Note. Numbers in parentheses indicate that the respective item parameters were unconstrained in the partial invariance model. The $\Delta \chi^{2}$ value for $D_{2}, D_{3}$, and $D_{4}$ was calculated as $k F_{k, \nu} . \boldsymbol{\Lambda}=$ loadings; $\boldsymbol{\tau}=$ intercepts; FIML = full-information maximum likelihood; $D_{2}, D_{3}, D_{4}=$ pooling methods. 
$D_{2}$

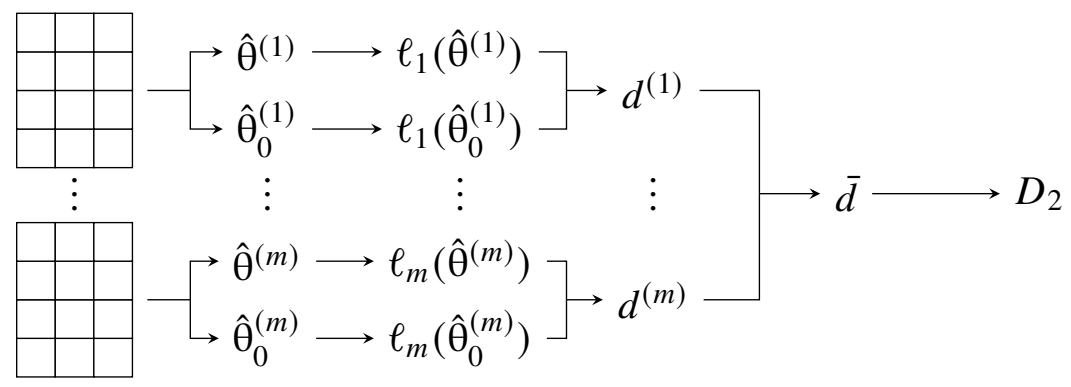

D

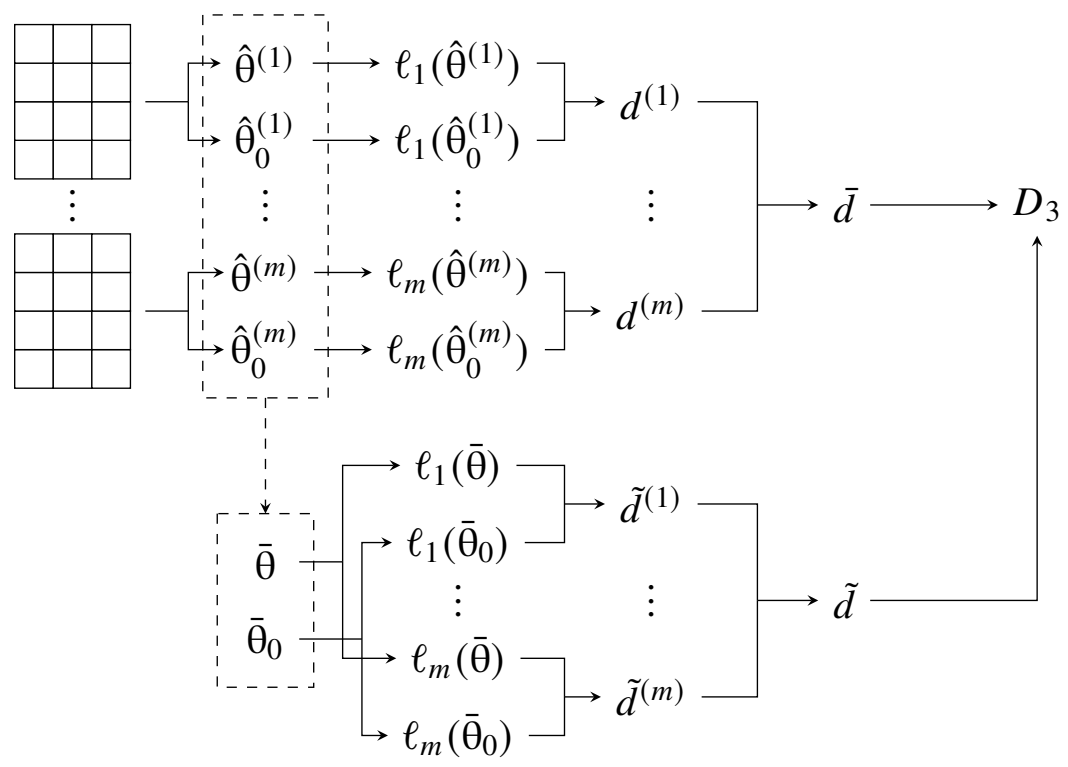

D

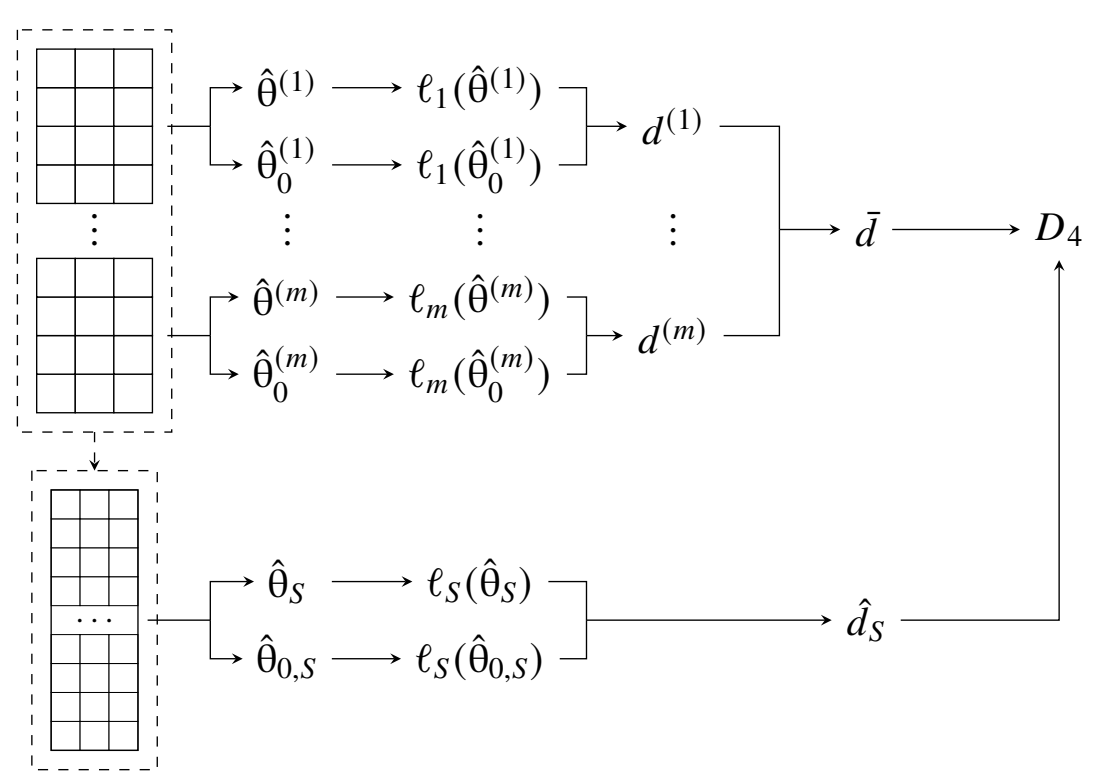

\section{Figure 1}

Schematic representation of pooling methods for LRTs under MI. The $D_{2}$ method is based only on the $\chi^{2}$ statistics obtained from the multiply imputed data, whereas $D_{3}$ also requires a reevaluation of the log-likelihood at the pooled parameter estimates, and $D_{4}$ requires an evaluation of the loglikelihood in a "stacked" data set. 

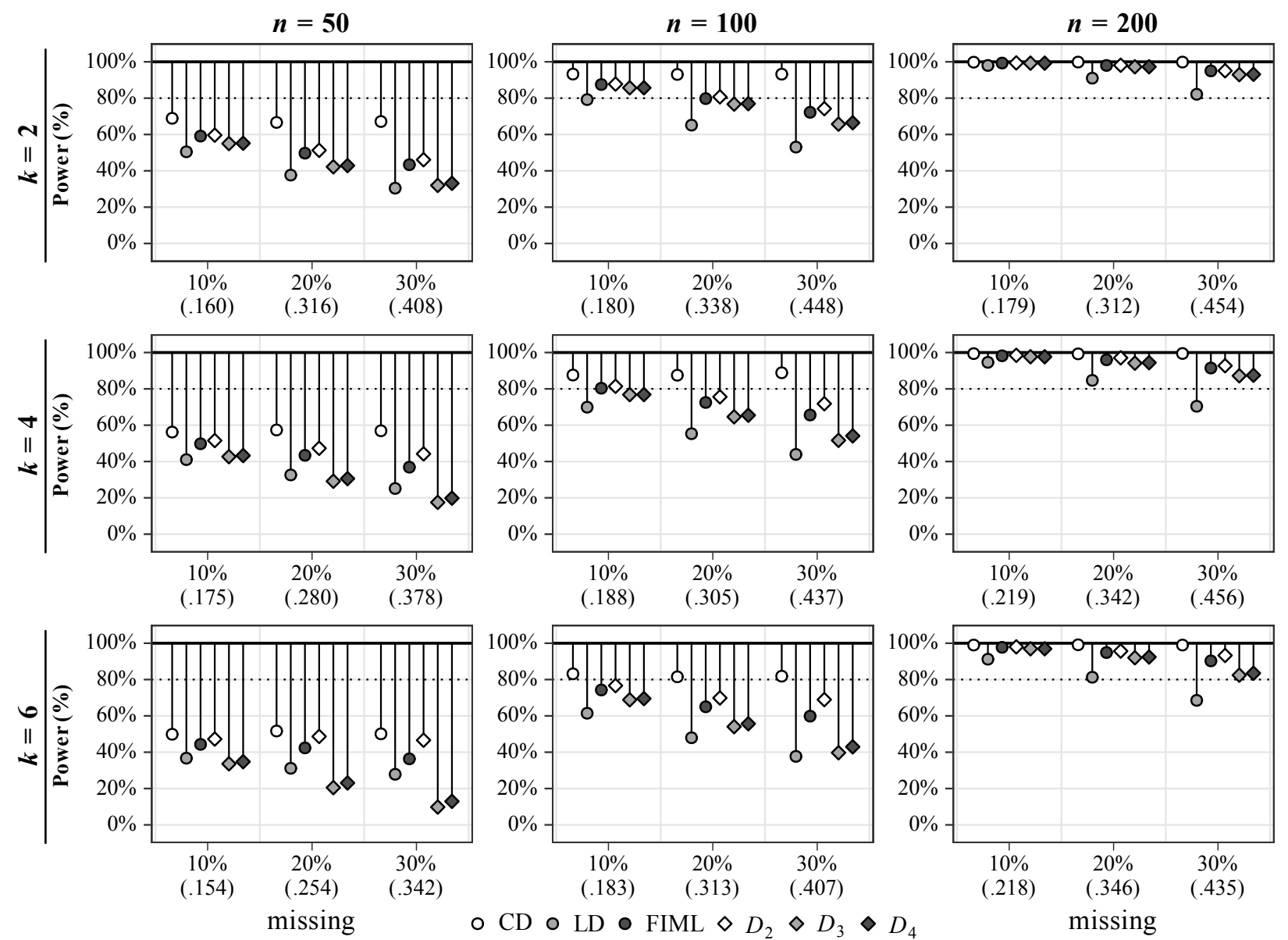

Figure 2

Power (in \%) in conditions with medium effect size $\left(R^{2}=.13\right)$ and MAR data in Study 1. Numbers in parentheses denote the empirical fraction of missing information per condition (FMI). $n=$ sample size; $k=$ number of predictors; $C D=$ complete data; $L D=$ listwise deletion; $F I M L=$ fullinformation maximum likelihood; $D_{2}, D_{3}, D_{4}=$ pooling methods. 

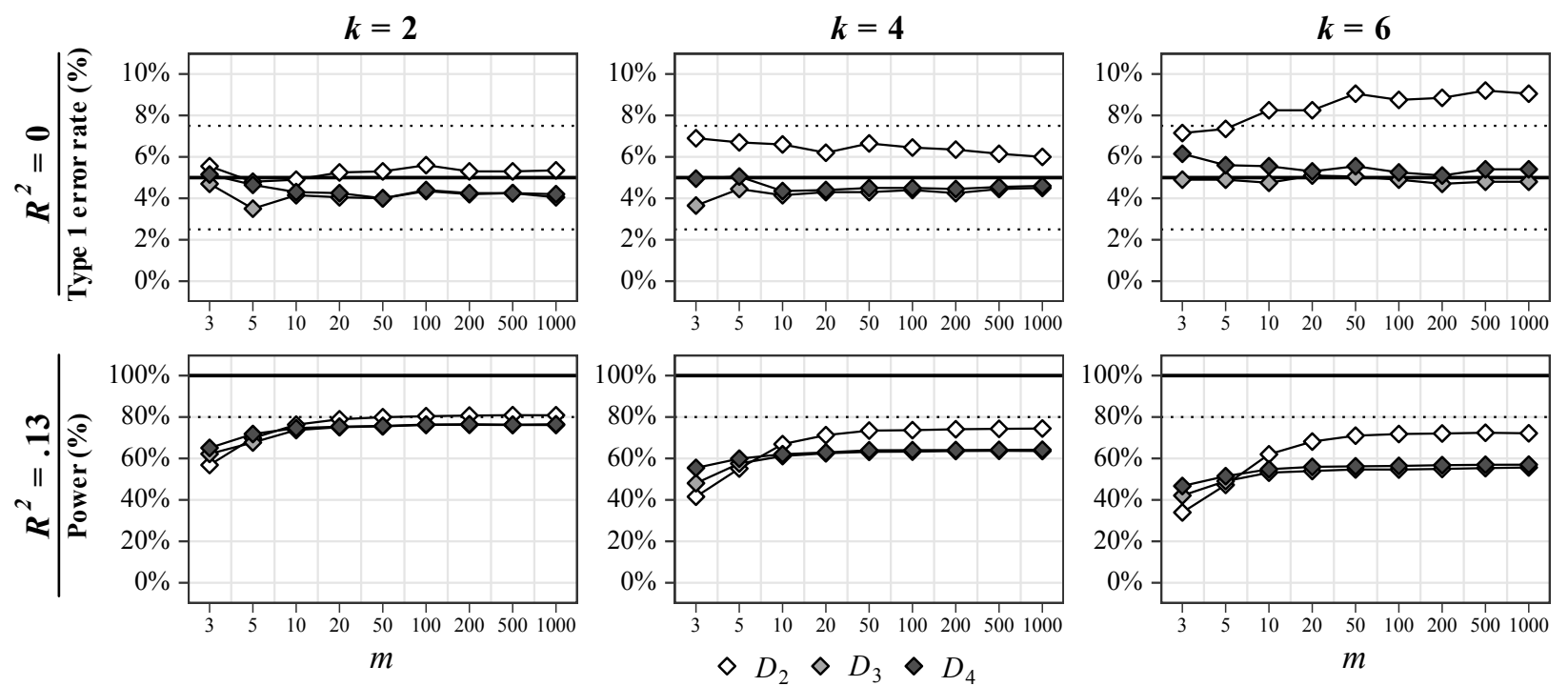

Figure 3

Type 1 error rates and power (in \%) in conditions with moderate sample size $(n=100)$ depending on the number of imputations $(m)$ in the context of Study 1. $k=$ number of predictors; $D_{2}, D_{3}$, $D_{4}=$ pooling methods. 

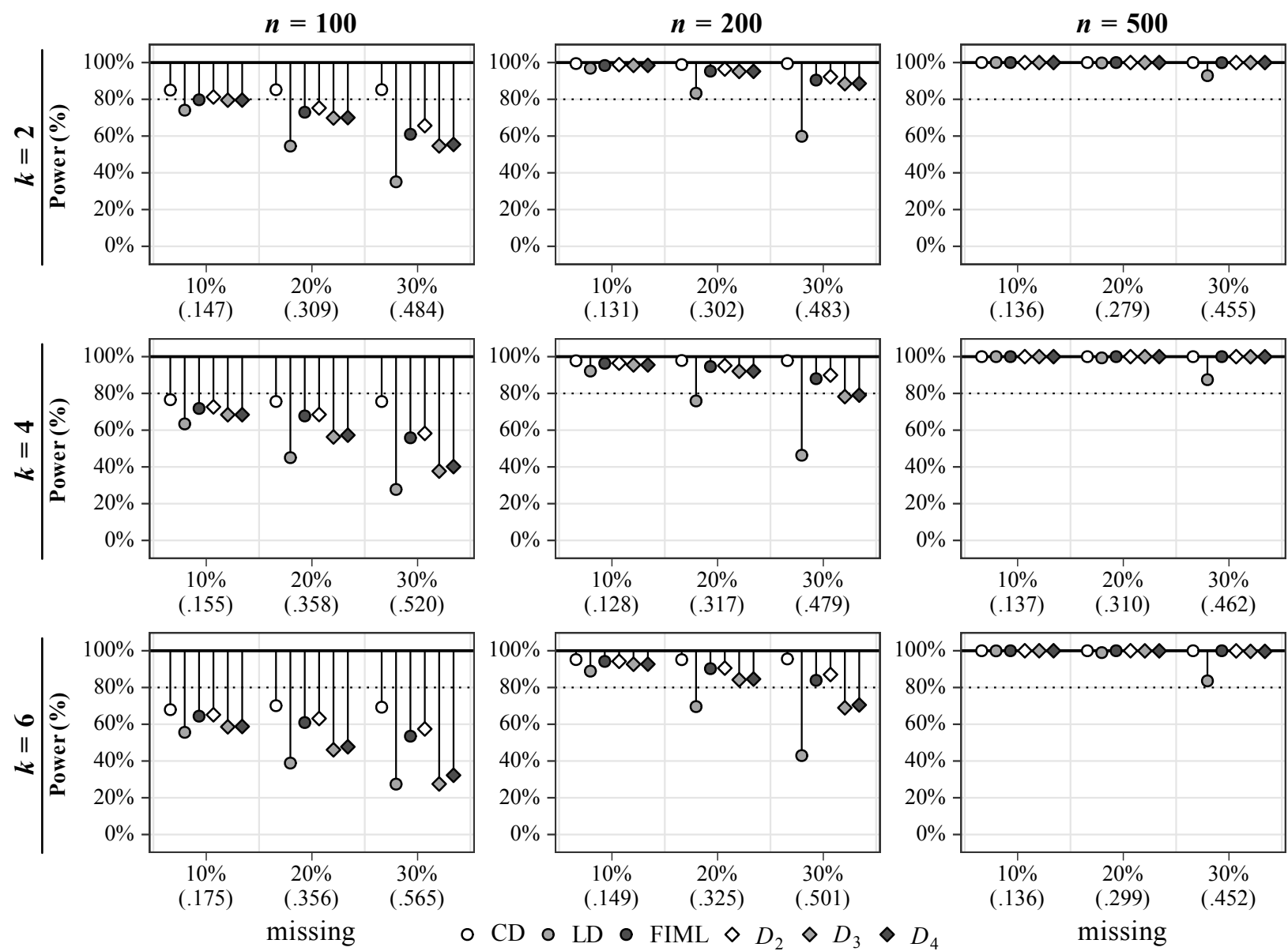

Figure 4

Power (in \%) in conditions with medium effect size $\left(R^{2}=.13\right)$ and MAR data in Study 2. Numbers in parentheses denote the empirical fraction of missing information per condition (FMI). $n=$ sample size; $k=$ number of predictors; $C D=$ complete data $; D=$ listwise deletion; $F I M L=$ fullinformation maximum likelihood; $D_{2}, D_{3}, D_{4}=$ pooling methods. 


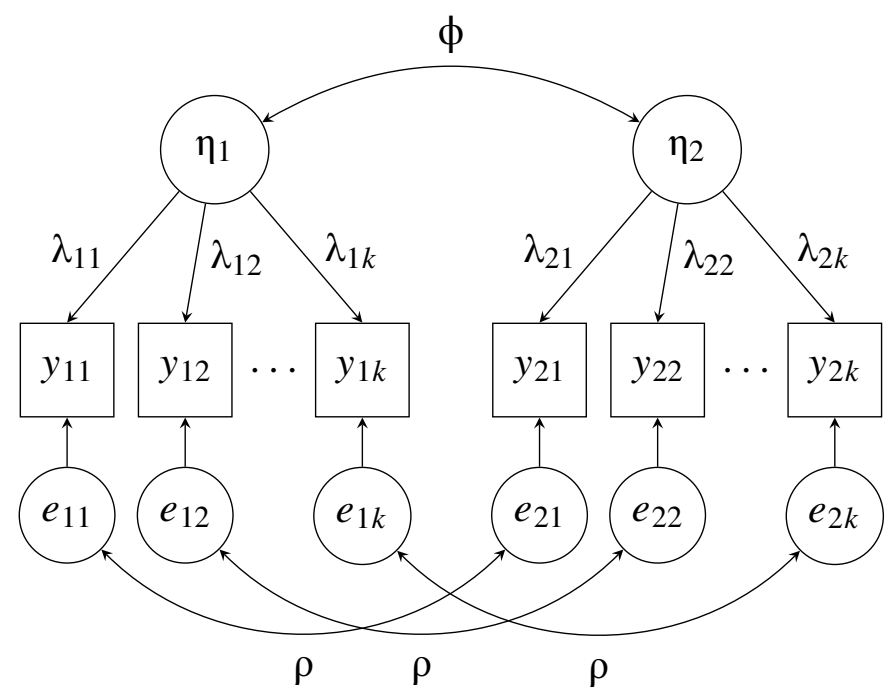

Figure 5

Data generating model in Study 3. 

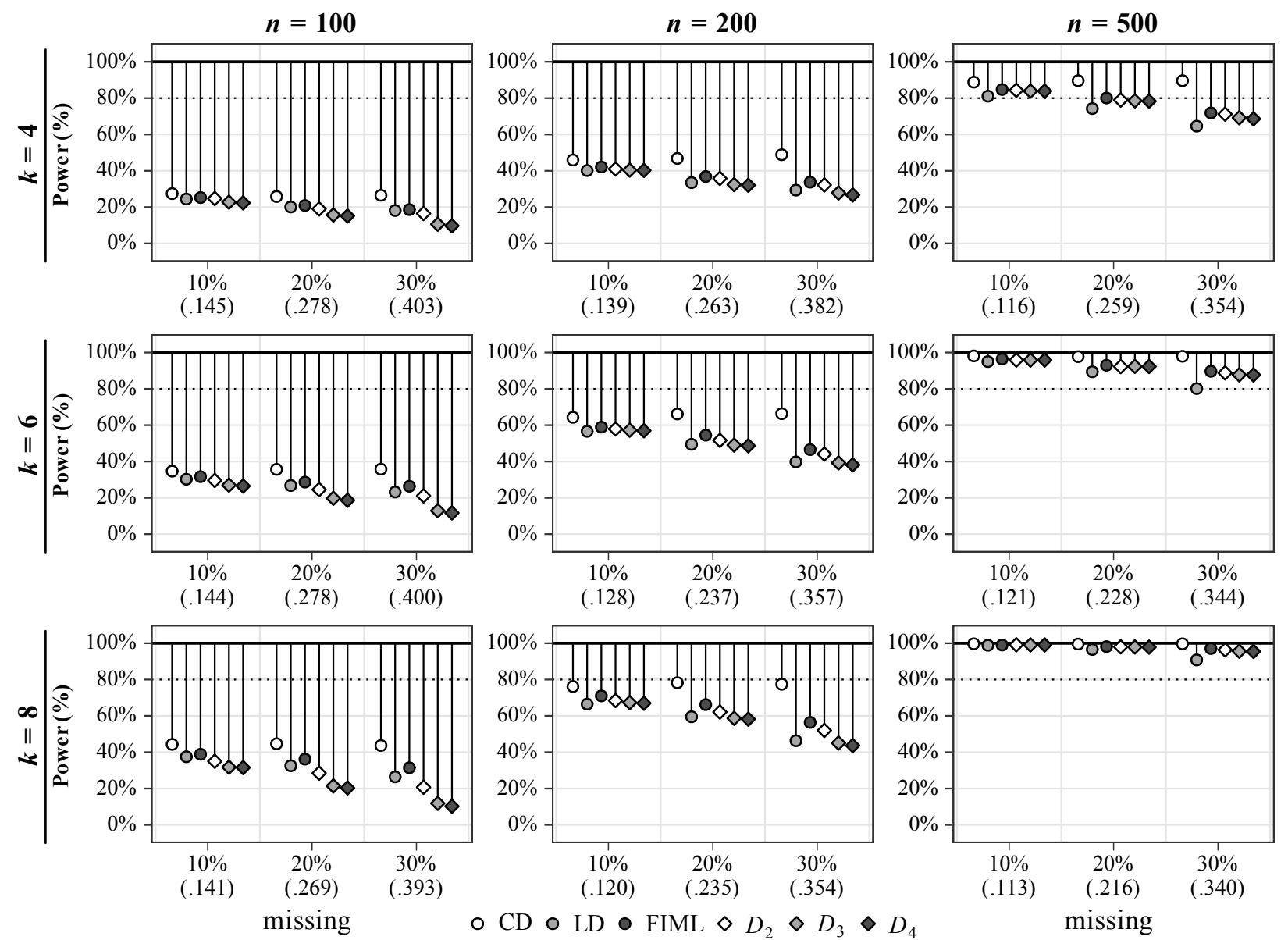

Figure 6

Power (in \%) in conditions with weak correlations between items $(\rho=.10)$ and MAR data in Study 3. Numbers in parentheses denote the empirical fraction of missing information per condition (FMI). $n=$ sample size; $k=$ number of items per factor; $C D=$ complete data; $L D=$ listwise deletion; FIML = full-information maximum likelihood; $D_{2}, D_{3}, D_{4}=$ pooling methods. 


\section{Appendix}

\section{Annotated Code for the Example Analysis}

In this example, we illustrate the key steps involved in conducting LRTs for the assessment of measurement invariance in the Example Analysis. The packages and data can be loaded as follows.

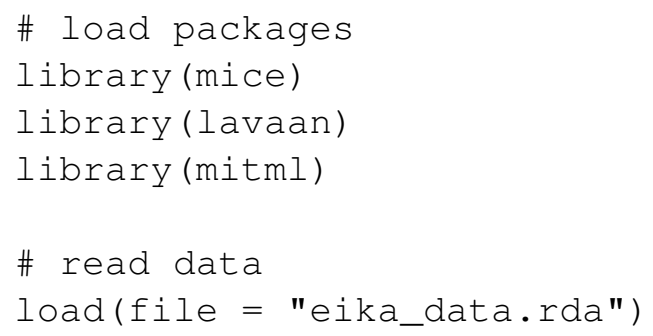

To impute the missing data, we use the mice ( ) command and save the results as a list:

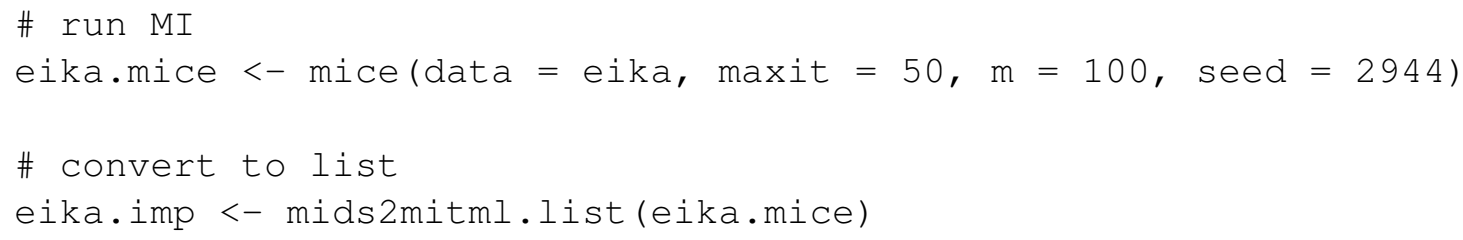

Given a list of imputed data sets, the first step to conducting the LRTs is to fit the configural, metric, and scalar invariance models to the imputed data. For example, the configural model is defined as:

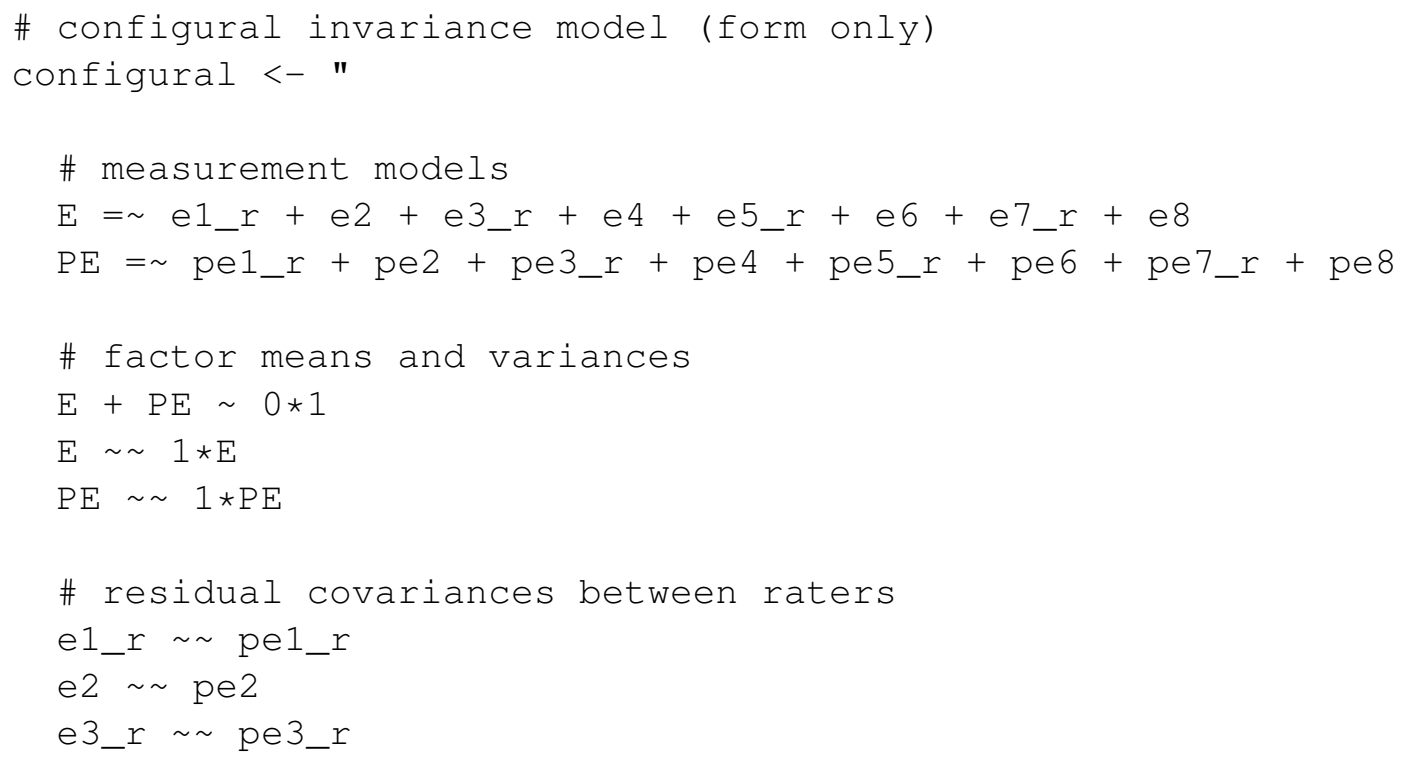




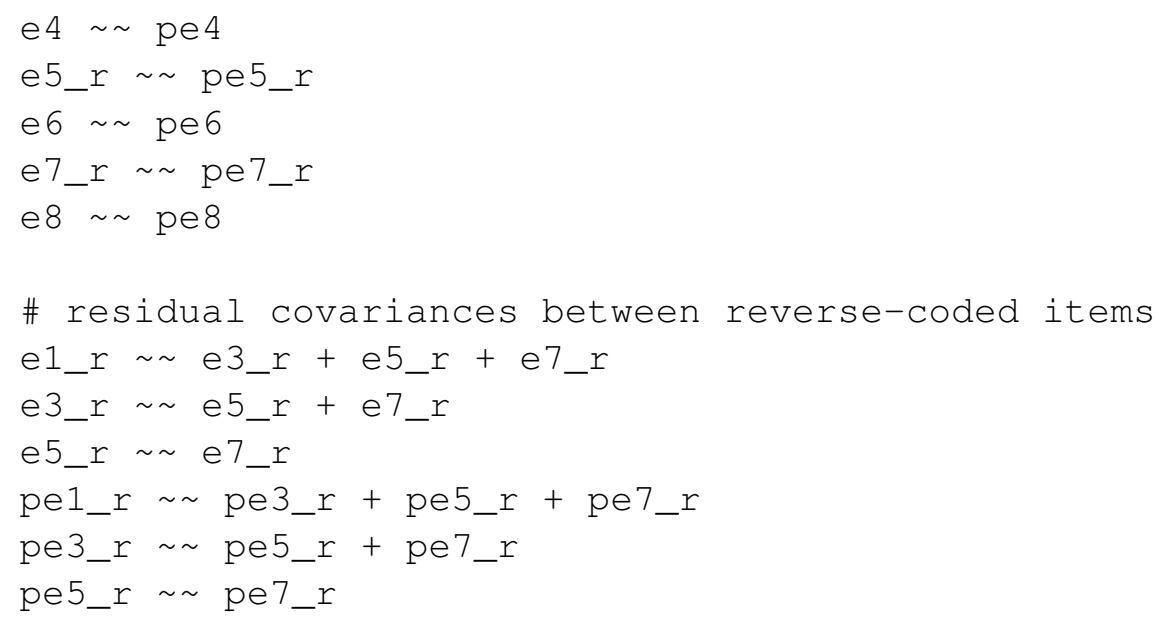

Once the models are defined, they can be fitted to the imputed data using the with ( ) and the cfa ( ) commands.

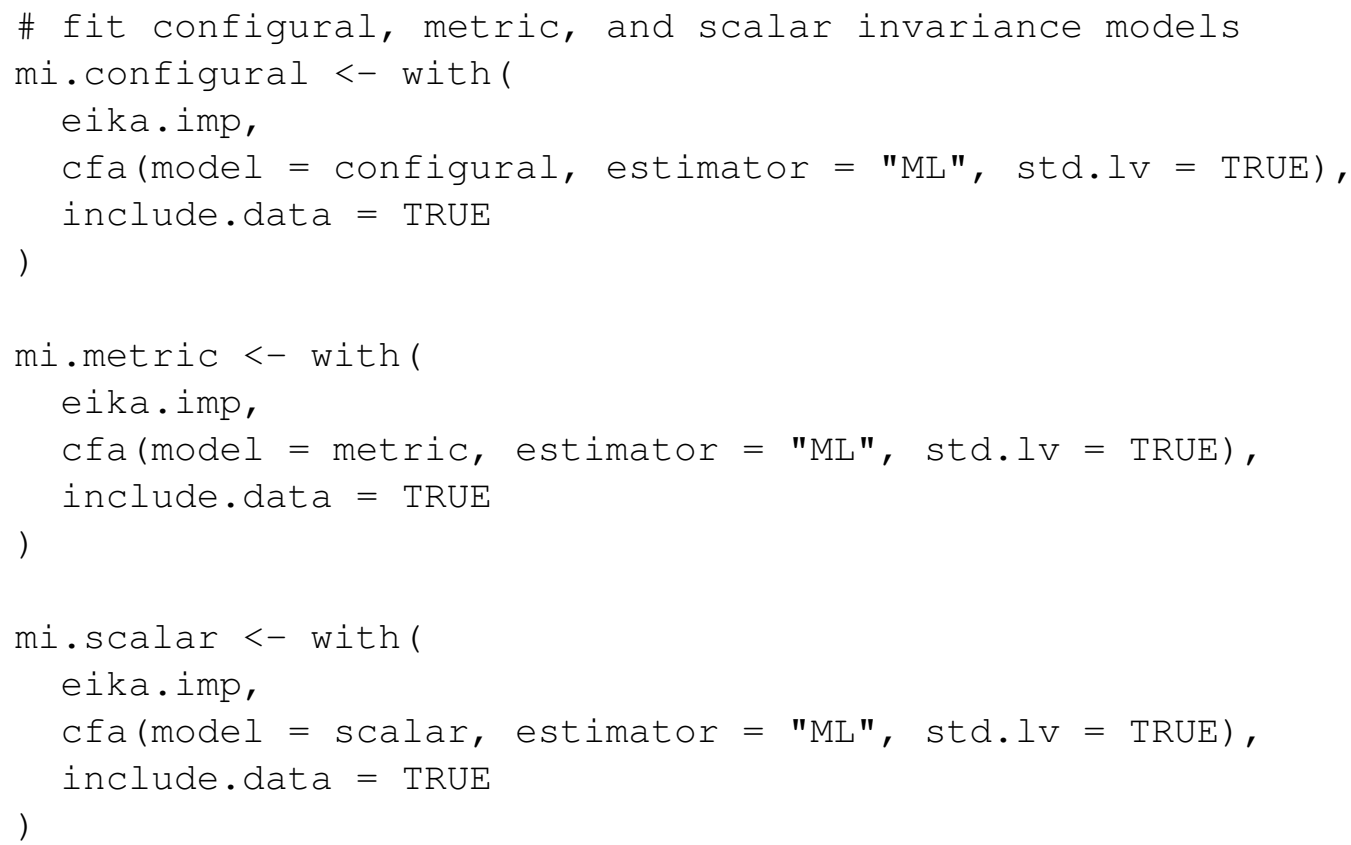

Then, the anova ( ) command can be used to compare the models with LRTs. This command supports the $D_{2}, D_{3}$, and $D_{4}$ methods. For example, to use $D_{4}$ to compare the configural, metric, and scalar invariance models, the command is as follows: 


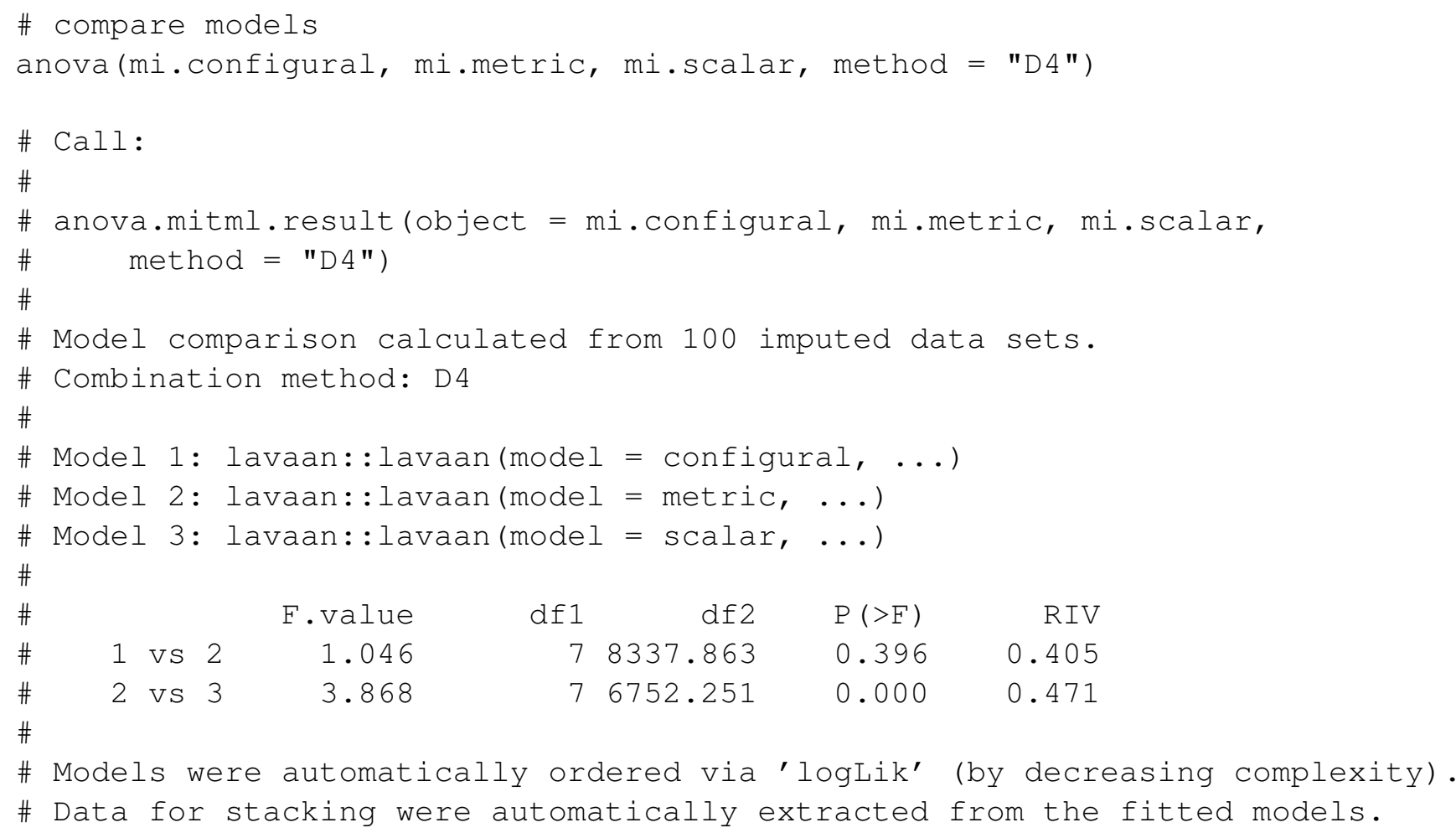

The output includes the results for the pooled LRTs with an $F$ reference distribution. Approximate $\chi^{2}$ values can be obtained by multiplying the $F$ values with the numerator degrees of freededom (see Table 5). 